\title{
Hölder regularity for degenerate parabolic obstacle problems
}

\author{
Verena Bögelein, Teemu Lukkari and Christoph Scheven
}

\begin{abstract}
We prove that weak solutions to the obstacle problem for the porous medium equation are locally Hölder continuous, provided that the obstacle is Hölder continuous.
\end{abstract}

\section{Introduction}

The porous medium equation

$$
\partial_{t} u-\Delta u^{m}=0
$$

PME for short, is an important prototype of a nonlinear parabolic equation. The name stems from modeling the flow of a gas in a porous medium. We restrict our attention to the slow diffusion case when $m>1$. In this case the equation is degenerate with respect to $u$, which means that the modulus of ellipticity vanishes when the solution is zero. This leads to interesting phenomena, for instance the existence of moving boundaries. The PME and its various generalizations have been extensively studied, and we refer to the monographs [7], [9], [17] and [18] for the basic theory and further references.

In the current paper we are interested in the obstacle problem for the PME. This problem can be fomulated as a variational inequality: formally, a function $u$ solves the obstacle problem with obstacle $\psi$ if $u \geq \psi$ and

$$
\int_{\Omega_{T}} \partial_{t} u\left(v^{m}-u^{m}\right)+\nabla u^{m} \cdot \nabla\left(v^{m}-u^{m}\right) d x d t \geq 0
$$

for all comparison maps $v$ such that $v \geq \psi$. A rigorous interpretation of the time term in this inequality requires some care as a solution might not have a time derivative

Key words and phrases: obstacle problem, porous medium equation, regularity. 2010 Mathematics Subject Classification: 35K65, 35B65, 35K86, 47J20. 
in a suitable sense, see [1] and [5] and Definition 2.1 below. The classical references for parabolic obstacle problems are [1], [15] and [16], and some of the more recent ones are [4], [5], [6], [12], [13] and [14]. However, most of them are dealing with the obstacle problem for the parabolic $p$-Laplacian equation. An alternative to the variational inequality is to define the solution to the obstacle problem to be the smallest supersolution lying above the obstacle, see [2] and [14] and Remark 2.3 below. We will not pursue the latter approach here.

The existence of appropriately defined weak solutions to the obstacle problem for the PME was shown in our previous paper [5]. These solutions belong to the class

$$
K_{\psi}\left(\Omega_{T}\right):=\left\{v \in C^{0}\left([0, T] ; L^{m+1}(\Omega)\right): v^{m} \in L^{2}\left(0, T ; H^{1}(\Omega)\right), v \geq \psi \text { a.e. on } \Omega_{T}\right\},
$$

where $\psi$ is a nonnegative obstacle function defined on a space-time cylinder $\Omega_{T}=$ $\Omega \times(0, T), \Omega$ is a bounded domain in $\mathbb{R}^{n}$, and $T>0$. Here our aim is to complement the results of [5] by establishing regularity for the solutions. The general guideline is that a solution to the obstacle problem should be as regular as a weak solution, as long as the regularity of the obstacle allows it. Hence a solution to the obstacle problem should be Hölder continuous if the obstacle is, since a weak solution to the PME is in general no better than Hölder continuous. This can be seen from explicit examples, such as the Barenblatt solution. In this respect, the following regularity result, which is the main result of this paper, is optimal.

Theorem 1.1. Suppose that the obstacle $\psi$ is Hölder continuous in $\Omega_{T}$, and let $u \in K_{\psi}\left(\Omega_{T}\right)$ be a local weak solution to the obstacle problem for the PME in the sense of Definition 2.1. Then $u$ is locally Hölder continuous in $\Omega_{T}$.

Remark 1.2. It is clear from the comparison with the obstacle-free case that the Hölder continuity of the solution can not be expected for arbitrary Hölder exponents $\alpha \in(0,1)$. In fact, our methods only yield Hölder continuity for some Hölder exponent that depends on $n, m$, and the Hölder exponent of the obstacle function, and is always smaller than the latter one. This is contrary to the results from [13] for obstacle problems related to parabolic $p$-Laplacian type equations, where the Hölder exponent of the solution turns out to be the same as the one of the obstacle function. However, the result in [13] relies on gradient bounds for solutions to $p$-Laplacian type equations, for which the analoga are not available in the case of the porous medium equation. In any case, the question for the optimal Hölder exponent in Theorem 1.1 remains an open problem.

The Hölder continuity of solutions to the porous medium equation in the obstacle-free case has first been established by DiBenedetto and Friedman [10]. The 
proof of the Hölder continuity is based on two main elements: energy estimates for truncations, and a De Giorgi type iteration argument to extract pointwise information from the energy estimates. The derivation of the energy estimates is intricate due to the necessarily complicated definition of weak solutions to the obstacle problem. For instance, the solution itself is not usually admissible as a comparison map in the variational inequality. In the iteration arguments, we need to construct cylinders with a proper scaling to balance the different powers in the energy estimates. The scaling is intrinsic, as it depends on the solution itself. This method has been introduced in [10] for the analysis of degenerate parabolic equations, cf. also [3], [8] and [9]. More precisely, in order to compensate for the inhomogeneous scaling of the underlying equation, we work with cylinders of the type

$$
Q_{\varrho, \theta \varrho^{2}}\left(x_{o}, t_{o}\right)=B_{\varrho}\left(x_{o}\right) \times\left(t_{o}-\theta \varrho^{2}, t_{o}\right),
$$

where the parameter $\theta$ is comparable to $u^{1-m}$. Additional care is needed in dealing with the degeneration of the porous medium equation, which occurs if the solution takes values close to zero in the sense that its infimum is considerably smaller than its supremum. This case, which we call the degenerate regime, requires a different treatment than the nondegenerate regime, in which the equation heuristically behaves like a linear equation with irregular coefficients. The proof is structured in such a way that both regimes are treated in a unified way whenever it is possible in order to work out the similarities and the differences of the two regimes.

The regularity of the obstacle enters the argument via a restriction on the truncation levels, which is needed to ensure that the test functions do not violate the obstacle condition.

The paper is organized as follows. In Section 2, we give the exact definition of a weak solution to the obstacle problem. In Section 3 we recall several technical results needed for the proofs. We prove the energy estimates for truncations in Section 4. These estimates are then used in Section 5 to prove local boundedness of solutions, and finally in Section 6 to prove the Hölder continuity.

\section{The obstacle problem}

In this section, we give the rigorous definition of a solution to the obstacle problem. For a bounded domain $\Omega \subset \mathbb{R}^{n}$ in dimension $n \in \mathbb{N}_{\geq 2}$ and a time $T>0$, we write $\Omega_{T}:=\Omega \times(0, T)$ for the space-time cylinder. We consider continuous obstacle functions $\psi \in C^{0}\left(\Omega_{T}, \mathbb{R}_{\geq 0}\right)$. For fixed $m>1$, we recall that the solution space is defined by

$$
K_{\psi}\left(\Omega_{T}\right):=\left\{v \in C^{0}\left([0, T] ; L^{m+1}(\Omega)\right): v^{m} \in L^{2}\left(0, T ; H^{1}(\Omega)\right), v \geq \psi \text { a.e. on } \Omega_{T}\right\} .
$$


Furthermore, the class of admissible comparison functions is

$$
K_{\psi}^{\prime}\left(\Omega_{T}\right):=\left\{v \in K_{\psi}\left(\Omega_{T}\right): \partial_{t}\left(v^{m}\right) \in L^{\frac{m+1}{m}}\left(\Omega_{T}\right)\right\} .
$$

Since the time derivative of a solution $u \in K_{\psi}\left(\Omega_{T}\right)$ to the obstacle problem might not exist in a sufficiently strong sense, we have to introduce a weak formulation of the time term in the variational inequality (1.1). To this end, we follow the approach by Alt \& Luckhaus [1] and define for every $u \in K_{\psi}\left(\Omega_{T}\right)$ and every comparison map $v \in K_{\psi}^{\prime}\left(\Omega_{T}\right)$

$$
\left\langle\left\langle\partial_{t} u, \alpha \eta\left(v^{m}-u^{m}\right)\right\rangle:=\int_{\Omega_{T}} \eta\left[\alpha^{\prime}\left[\frac{1}{m+1} u^{m+1}-u v^{m}\right]-\alpha u \partial_{t} v^{m}\right] d z,\right.
$$

where $\alpha \in W_{0}^{1, \infty}\left([0, T], \mathbb{R}_{\geq 0}\right)$ and $\eta \in C_{0}^{1}\left(\Omega, \mathbb{R}_{\geq 0}\right)$ denote cut-off functions in time, respectively in space.

We are now in a position to define the notion of a local weak solution to the obstacle problem.

Definition 2.1. A nonnegative function $u \in K_{\psi}\left(\Omega_{T}\right)$ is a local weak solution to the obstacle problem for the porous medium equation if and only if

$$
\left\langle\left\langle\partial_{t} u, \alpha \eta\left(v^{m}-u^{m}\right)\right\rangle+\int_{\Omega_{T}} \alpha \nabla u^{m} \cdot \nabla\left(\eta\left(v^{m}-u^{m}\right)\right) d z \geq 0\right.
$$

holds true for all comparison maps $v \in K_{\psi}^{\prime}\left(\Omega_{T}\right)$, every cut-off function in time $\alpha \in$ $W_{0}^{1, \infty}\left([0, T], \mathbb{R}_{\geq 0}\right)$ and every cut-off function in space $\eta \in C_{0}^{1}\left(\Omega, \mathbb{R}_{\geq 0}\right)$.

Remark 2.2. Since $u \in C^{0}\left([0, T] ; L^{m+1}(\Omega)\right)$, this definition is consistent with the notion of weak solution used in [5, Def. 2.1]. This means that for more general cut-off functions in time with $\alpha(0) \neq 0$, the above notion of solution implies

$$
\left\langle\left\langle\partial_{t} u, \alpha \eta\left(v^{m}-u^{m}\right)\right\rangle\right\rangle_{u_{o}}+\int_{\Omega_{T}} \alpha \nabla u^{m} \cdot \nabla\left(\eta\left(v^{m}-u^{m}\right)\right) d z \geq 0
$$

for $u_{o}:=u(\cdot, 0)$ if we define

$$
\begin{aligned}
\left\langle\left\langle\partial_{t} u, \alpha \eta\left(v^{m}-u^{m}\right)\right\rangle\right\rangle_{u_{o}}:= & \int_{\Omega_{T}} \eta\left[\alpha^{\prime}\left[\frac{1}{m+1} u^{m+1}-u v^{m}\right]-\alpha u \partial_{t} v^{m}\right] d z \\
& +\alpha(0) \int_{\Omega} \eta\left[\frac{1}{m+1} u_{o}^{m+1}-u_{o} v^{m}(\cdot, 0)\right] d x .
\end{aligned}
$$

For obstacles $\psi$ with

$$
\psi^{m} \in L^{2}\left(0, T ; H^{1}(\Omega)\right), \quad \partial_{t}\left(\psi^{m}\right) \in L^{\frac{m+1}{m}}\left(\Omega_{T}\right) \quad \text { and } \quad \psi^{m}(\cdot, 0) \in H^{1}(\Omega),
$$

an existence result for local weak solutions is contained in our earlier work [5]. 
Moreover, from [5, Lemma 3.2] we know that a local weak solution with $\partial_{t} u \in$ $L^{2}\left(0, T ; H^{-1}(\Omega)\right)$ is also a local strong solution in the sense that for all comparison maps $v \in K_{\psi}\left(\Omega_{T}\right)$, every cut-off function in time $\alpha \in W^{1, \infty}\left([0, T], \mathbb{R}_{\geq 0}\right)$ with $\alpha(T)=0$ and every cut-off function in space $\eta \in C_{0}^{1}\left(\Omega, \mathbb{R}_{\geq 0}\right)$ we have

$$
\int_{0}^{T}\left\langle\partial_{t} u, \alpha \eta\left(v^{m}-u^{m}\right)\right\rangle d t+\int_{\Omega_{T}} \alpha \nabla u^{m} \cdot \nabla\left(\eta\left(v^{m}-u^{m}\right)\right) d z \geq 0
$$

where here, $\langle\cdot, \cdot\rangle$ denotes the dual pairing between $H^{-1}(\Omega)$ and $H_{0}^{1}(\Omega)$.

Remark 2.3. An alternative to the variational inequality described above is to define the solution to the obstacle problem to be the smallest weak supersolution lying above the obstacle $\psi$. This approach is used in [14] in a nonlinear parabolic setting, and in [2] for the PME. It is analogous to the balayage concept of classical potential theory.

Existence and uniqueness of the smallest supersolution follow quite easily from the definitions. However, the connection between the smallest supersolution and the variational solutions studied here is less clear. In this direction, we have an approximation property for the smallest supersolution: for continuous compactly supported obstacles, the smallest supersolution is a pointwise limit of variational solutions. See [2] for the proof. The approximation property together with a stability result from [5] implies that the smallest supersolution is also a variational solution for sufficiently smooth obstacles. Further, since our Hölder estimate depends on the obstacle only via its Hölder norm, Theorem 1.1 holds also for the smallest supersolution.

The question whether all variational solutions are also smallest supersolutions remains a very interesting open problem.

\section{Preliminaries}

\subsection{Notation}

We use the notation $Q_{\varrho, \theta}\left(z_{o}\right):=B_{\varrho}\left(x_{o}\right) \times\left(t_{o}-\theta, t_{o}\right) \subset \mathbb{R}^{n+1}$ for backward parabolic cylinders, where the point $z_{o}=\left(x_{o}, t_{o}\right) \in \mathbb{R}^{n+1}$ is the vertex of the cylinder, and $\varrho, \theta>0$.

\subsection{Auxiliary material}

For later reference, we recall the parabolic version of the Gagliardo-Nirenberg inequality. 
Lemma 3.1. Let $Q_{\varrho, \theta}\left(z_{o}\right) \subset \mathbb{R}^{n+1}$ be a parabolic cylinder and $1<p, r<\infty$. For every

$$
u \in L^{\infty}\left(t_{o}-\theta, t_{o} ; L^{r}\left(B_{\varrho}\left(x_{o}\right)\right)\right) \cap L^{p}\left(t_{o}-\theta, t_{o} ; W^{1, p}\left(B_{\varrho}\left(x_{o}\right)\right)\right)
$$

we have $u \in L^{q}\left(Q_{\varrho, \theta}\left(z_{o}\right)\right)$ for $q=p\left(1+\frac{r}{n}\right)$ with the estimate

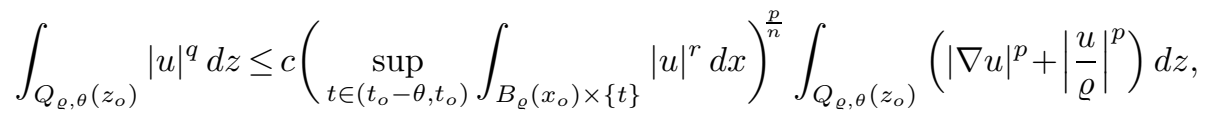

where $c=c(n, p, r)$.

The proof of the following well-known lemma can be found e.g. in [11, Lemma 7.1].

Lemma 3.2. Let $\left(X_{i}\right)_{i \in \mathbb{N}_{0}}$ be a sequence of positive real numbers with

$$
X_{i+1} \leq C B^{i} X_{i}^{1+\alpha} \quad \text { for all } i \in \mathbb{N}_{0},
$$

for constants $C, \alpha>0$ and $B>1$. Then

$$
X_{0} \leq C^{-\frac{1}{\alpha}} B^{-\frac{1}{\alpha^{2}}}
$$

implies $X_{i} \rightarrow 0$ as $i \rightarrow \infty$.

We will also use DeGiorgi's isoperimetric inequality. See e.g. [9, §2, Lemma 2.2] for the proof.

Lemma 3.3. Let $v \in W^{1,1}\left(B_{\varrho}\left(x_{o}\right)\right)$, and let $k<\ell$ be real numbers. There exists a constant $c=c(n)$, such that

$$
(\ell-k)\left|B_{\varrho}\left(x_{o}\right) \cap\{v>\ell\}\right| \leq \frac{c \varrho^{n+1}}{\left|B_{\varrho}\left(x_{o}\right) \cap\{v<k\}\right|} \int_{B_{\varrho}\left(x_{o}\right) \cap\{k<v<\ell\}}|\nabla v| d x .
$$

\subsection{Mollification in time}

In order to deal with the possible lack of differentiability in time of weak solutions, the following time mollification of functions $v: \Omega_{T} \rightarrow \mathbb{R}$ has proved to be useful.

$$
\llbracket v \rrbracket_{h}(x, t):=\frac{1}{h} \int_{0}^{t} e^{\frac{s-t}{h}} v(x, s) d s .
$$

In the following lemma, we list some elementary properties of this mollification that will be needed in the proof later (cf. [16]). 
Lemma 3.4. Let $p \geq 1$.

(i) If $v \in L^{p}\left(\Omega_{T}\right)$ then $\llbracket v \rrbracket_{h} \rightarrow v$ in $L^{p}\left(\Omega_{T}\right)$ as $h \downarrow 0$ and

$$
\partial_{t} \llbracket v \rrbracket_{h}=\frac{1}{h}\left(v-\llbracket v \rrbracket_{h}\right) \in L^{p}\left(\Omega_{T}\right) \quad \text { for every } h>0 .
$$

(ii) If $\nabla v \in L^{p}\left(\Omega_{T}, \mathbb{R}^{n}\right)$ then $\nabla \llbracket v \rrbracket_{h}=\llbracket \nabla v \rrbracket_{h}$ and $\nabla \llbracket v \rrbracket_{h} \rightarrow \nabla v$ in $L^{p}\left(\Omega_{T}, \mathbb{R}^{n}\right)$ as $h \downarrow 0$.

(iii) If $v \in C^{0}\left(\overline{\Omega_{T}}\right)$, then $\llbracket v \rrbracket_{h} \rightarrow v$ uniformly in $\Omega_{T}$ as $h \downarrow 0$.

\section{Energy estimates}

\subsection{Caccioppoli type estimates}

Here, we derive energy estimates for the truncated functions

$$
\left[u^{m}-k^{m}\right]_{+}:=\max \left\{u^{m}-k^{m}, 0\right\} \quad \text { and } \quad\left[u^{m}-k^{m}\right]_{-}:=\max \left\{k^{m}-u^{m}, 0\right\},
$$

where $k>0$ denotes a constant.

Lemma 4.1. Let $Q_{1}:=Q_{\varrho_{1}, \theta_{1}}\left(z_{o}\right) \Subset \Omega_{T}$ and $Q_{2}:=Q_{\varrho_{2}, \theta_{2}}\left(z_{o}\right) \subset Q_{1}$ be two cylinders with $\varrho_{2}<\varrho_{1}, \theta_{2}<\theta_{1}$, and assume that $\psi \in C^{0}\left(\Omega_{T}, \mathbb{R}_{\geq 0}\right)$. Then, for every local weak solution $u \in K_{\psi}\left(\Omega_{T}\right)$ to the obstacle problem for the porous medium equation in the sense of Definition 2.1, we have the following estimates.

(i) For every $k \geq \sup _{Q_{1}} \psi$ we have

$$
\begin{aligned}
& \sup _{t \in\left(t_{o}-\theta_{2}, t_{o}\right)} \int_{B_{\varrho_{2}}\left(x_{o}\right) \times\{t\}} u^{1-m}\left[u^{m}-k^{m}\right]_{+}^{2} d x+\int_{Q_{2}}\left|\nabla\left[u^{m}-k^{m}\right]_{+}\right|^{2} d z \\
& \leq c(m)\left(\frac{1}{\left(\varrho_{1}-\varrho_{2}\right)^{2}}+\frac{k^{1-m}}{\theta_{1}-\theta_{2}}\right) \int_{Q_{1}}\left[u^{m}-k^{m}\right]_{+}^{2} d z .
\end{aligned}
$$

(ii) For every $k>0$, we have

$$
\begin{aligned}
\sup _{t \in\left(t_{o}-\theta_{2}, t_{o}\right)} & k^{1-m} \int_{B_{\varrho_{2}}\left(x_{o}\right) \times\{t\}}\left[u^{m}-k^{m}\right]_{-}^{2} d x+\int_{Q_{2}}\left|\nabla\left[u^{m}-k^{m}\right]_{-}\right|^{2} d z \\
\leq & \frac{c(m)}{\left(\varrho_{1}-\varrho_{2}\right)^{2}} \int_{Q_{1}}\left[u^{m}-k^{m}\right]_{-}^{2} d z+\frac{c(m)}{\theta_{1}-\theta_{2}} \int_{Q_{1}} \int_{0}^{\left[u^{m}-k^{m}\right]_{-}}\left(k^{m}-\tau\right)^{\frac{1-m}{m}} \tau d \tau d z .
\end{aligned}
$$

Proof of $(i)$. By restricting ourselves to a compact subdomain of $\Omega_{T}$ if necessary, we may assume $\psi \in C^{0}\left(\overline{\Omega_{T}}\right)$, so that Lemma 3.4 (iii) is applicable to $v=\psi$. Moreover, we assume $z_{o}=0$ for notational convenience. We choose a cut-off function $\alpha \in W_{0}^{1, \infty}\left(\left[-\theta_{1}, 0\right], \mathbb{R}_{\geq 0}\right)$ and $\eta \in C_{0}^{1}\left(B_{\varrho_{1}}, \mathbb{R}_{\geq 0}\right)$. In the variational inequality (2.2), we choose

$$
v_{h}^{m}:=\llbracket u^{m} \rrbracket_{h}-\left(\llbracket u^{m} \rrbracket_{h}-k^{m}\right)_{+}+\left\|\psi^{m}-\llbracket \psi^{m} \rrbracket_{h}\right\|_{L^{\infty}\left(\Omega_{T}\right)}
$$


as comparison map for $u$, for some $h>0$, with the mollification $\llbracket \cdot \rrbracket_{h}$ as defined in (3.1). This map satisfies

$$
\partial_{t} v_{h}^{m}=\partial_{t} \min \left\{\llbracket u^{m} \rrbracket_{h}, k^{m}\right\} \in L^{\frac{m+1}{m}}\left(\Omega_{T}\right) .
$$

The comparison map $v_{h}$ is admissible because we have

$$
v_{h}^{m}=\min \left\{\llbracket u^{m} \rrbracket_{h}, k^{m}\right\}+\left\|\psi^{m}-\llbracket \psi^{m} \rrbracket_{h}\right\|_{L^{\infty}\left(\Omega_{T}\right)} \geq \psi^{m} \quad \text { on } Q_{1},
$$

since $u \geq \psi$ a.e. on $\Omega_{T}$ and $k \geq \sup _{Q_{1}} \psi$ by assumption. We note that it is sufficient to check the obstacle condition for $v_{h}$ on $\operatorname{supp}(\alpha \eta) \subset Q_{1}$. We therefore know

$$
\mathrm{I}_{h}+\mathrm{II}_{h}:=\left\langle\left\langle\partial_{t} u, \alpha \eta^{2}\left(v_{h}^{m}-u^{m}\right)\right\rangle\right\rangle+\int_{Q_{1}} \alpha \nabla u^{m} \cdot \nabla\left(\eta^{2}\left(v_{h}^{m}-u^{m}\right)\right) d z \geq 0 .
$$

For the estimate of $\mathrm{I}_{h}$, we use Lemma 3.4(i) to calculate

$$
\begin{aligned}
& \int_{Q_{1}} \eta^{2} \alpha u \partial_{t} v_{h}^{m} d z \\
&=\int_{Q_{1}} \eta^{2} \alpha \llbracket u^{m} \rrbracket_{h}^{\frac{1}{m}} \partial_{t} v_{h}^{m} d z+\int_{Q_{1} \cap\left\{\llbracket u^{m} \rrbracket_{h} \leq k^{m}\right\}} \eta^{2} \alpha\left(u-\llbracket u^{m} \rrbracket_{h}^{\frac{1}{m}}\right) \frac{1}{h}\left(u^{m}-\llbracket u^{m} \rrbracket h\right) d z \\
& \geq \int_{Q_{1}} \eta^{2} \alpha \llbracket u^{m} \rrbracket_{h}^{\frac{1}{m}} \partial_{t}\left(\llbracket u^{m} \rrbracket_{h}-\left(\llbracket u^{m} \rrbracket_{h}-k^{m}\right)_{+}\right) d z \\
&=\int_{Q_{1}} \eta^{2}\left(\alpha \frac{m}{m+1} \partial_{t} \llbracket u^{m} \rrbracket_{h}^{\frac{m+1}{m}}+\alpha^{\prime} \llbracket u^{m} \rrbracket_{h}^{\frac{1}{m}}\left(\llbracket u^{m} \rrbracket_{h}-k^{m}\right)_{+}\right) d z \\
&+\int_{Q_{1}} \eta^{2} \alpha \partial_{t} \llbracket u^{m} \rrbracket_{h}^{\frac{1}{m}}\left(\llbracket u^{m} \rrbracket_{h}-k^{m}\right)_{+} d z .
\end{aligned}
$$

The last integrand can be re-written using the identity

$$
\partial_{t} \llbracket u^{m} \rrbracket_{h}^{\frac{1}{m}}\left(\llbracket u^{m} \rrbracket_{h}-k^{m}\right)_{+}=\frac{1}{m} \frac{\partial}{\partial t} \int_{0}^{\left(\llbracket u^{m} \rrbracket_{h}-k^{m}\right)_{+}}\left(k^{m}+\tau\right)^{\frac{1-m}{m}} \tau d \tau .
$$

Plugging this into the preceding inequality and integrating by parts, we deduce

$$
\begin{aligned}
\int_{Q_{1}} \eta^{2} \alpha u \partial_{t} v_{h}^{m} d z \geq & \int_{Q_{1}} \eta^{2} \alpha^{\prime}\left(-\frac{m}{m+1} \llbracket u^{m} \rrbracket_{h}^{\frac{m+1}{m}}+\llbracket u^{m} \rrbracket_{h}^{\frac{1}{m}}\left(\llbracket u^{m} \rrbracket_{h}-k^{m}\right)_{+}\right) d z \\
& -\frac{1}{m} \int_{Q_{1}} \eta^{2} \alpha^{\prime} \int_{0}^{\left(\llbracket u^{m} \rrbracket_{h}-k^{m}\right)_{+}}\left(k^{m}+\tau\right)^{\frac{1-m}{m}} \tau d \tau d z .
\end{aligned}
$$


Recalling definition (2.1) we deduce

$$
\begin{aligned}
\mathrm{I}_{h}= & \int_{Q_{1}} \eta^{2}\left[\alpha^{\prime}\left[\frac{1}{m+1} u^{m+1}-u v_{h}^{m}\right]-\alpha u \partial_{t} v_{h}^{m}\right] d z \\
\leq & \int_{Q_{1}} \eta^{2} \alpha^{\prime}\left(\frac{1}{m+1} u^{m+1}-u\left(\llbracket u^{m} \rrbracket_{h}-\left(\llbracket u^{m} \rrbracket_{h}-k^{m}\right)_{+}+\left\|\psi^{m}-\llbracket \psi^{m} \rrbracket_{h}\right\|_{L^{\infty}}\right) d z\right. \\
& \left.+\int_{Q_{1}} \eta^{2} \alpha^{\prime} \frac{m}{m+1} \llbracket u^{m} \rrbracket_{h}^{\frac{m+1}{m}}-\llbracket u^{m} \rrbracket_{h}^{\frac{1}{m}}\left(\llbracket u^{m} \rrbracket_{h}-k^{m}\right)_{+}\right) d z \\
& +\frac{1}{m} \int_{Q_{1}} \eta^{2} \alpha^{\prime} \int_{0}^{\left(\llbracket u^{m} \rrbracket_{h}-k^{m}\right)_{+}}\left(k^{m}+\tau\right)^{\frac{1-m}{m}} \tau d \tau d z .
\end{aligned}
$$

Lemma 3.4 implies $\llbracket u^{m} \rrbracket_{h} \rightarrow u^{m}$ in $L^{\frac{m+1}{m}}\left(Q_{1}\right)$ as well as $\left\|\psi^{m}-\llbracket \psi^{m} \rrbracket_{h}\right\|_{L^{\infty}} \rightarrow 0$ as $h \downarrow 0$. As a consequence, the sum of the first two integrals on the right-hand side vanishes in the limit $h \downarrow 0$ and we infer

$$
\underset{h \downarrow 0}{\limsup } \mathrm{I}_{h} \leq \frac{1}{m} \int_{Q_{1}} \eta^{2} \alpha^{\prime} \int_{0}^{\left[u^{m}-k^{m}\right]_{+}}\left(k^{m}+\tau\right)^{\frac{1-m}{m}} \tau d \tau d z .
$$

Next, we note that $\nabla\left(\eta^{2}\left(v_{h}^{m}-u^{m}\right)\right) \rightarrow-\nabla\left(\eta^{2}\left[u^{m}-k^{m}\right]_{+}\right)$in $L^{2}\left(Q_{1}\right)$ as $h \downarrow 0$ by Lemma 3.4. Using moreover the identity

$$
\nabla u^{m}=\nabla\left[u^{m}-k^{m}\right]_{+} \quad \text { a.e. on } \Omega_{T} \cap\{u \geq k\} \text {, }
$$

we calculate

$$
\begin{aligned}
\lim _{h \downarrow 0} \mathrm{II}_{h}= & -\int_{Q_{1}} \alpha \nabla u^{m} \cdot \nabla\left(\eta^{2}\left[u^{m}-k^{m}\right]_{+}\right) d z \\
= & -\int_{Q_{1}} \alpha \eta^{2}\left|\nabla\left[u^{m}-k^{m}\right]_{+}\right|^{2} d z \\
& -2 \int_{Q_{1}} \alpha \eta \nabla\left[u^{m}-k^{m}\right]_{+} \cdot \nabla \eta\left[u^{m}-k^{m}\right]_{+} d z \\
\leq & -\frac{1}{2} \int_{Q_{1}} \alpha \eta^{2}\left|\nabla\left[u^{m}-k^{m}\right]_{+}\right|^{2} d z+2 \int_{Q_{1}} \alpha|\nabla \eta|^{2}\left[u^{m}-k^{m}\right]_{+}^{2} d z .
\end{aligned}
$$

Combining (4.3) and (4.5) with (4.1), we arrive at

$$
\begin{aligned}
& \frac{1}{2} \int_{Q_{1}} \alpha \eta^{2}\left|\nabla\left[u^{m}-k^{m}\right]_{+}\right|^{2} d z \\
& \leq \frac{1}{m} \int_{Q_{1}} \eta^{2} \alpha^{\prime} \int_{0}^{\left[u^{m}-k^{m}\right]_{+}}\left(k^{m}+\tau\right)^{\frac{1-m}{m}} \tau d \tau d z+2 \int_{Q_{1}} \alpha|\nabla \eta|^{2}\left[u^{m}-k^{m}\right]_{+}^{2} d z
\end{aligned}
$$


Now, we choose $\eta \in C_{0}^{1}\left(B_{\varrho_{1}}, \mathbb{R}_{\geq 0}\right)$ with $\eta \equiv 1$ on $B_{\varrho_{2}}$ and $|\nabla \eta| \leq \frac{2}{\varrho_{1}-\varrho_{2}}$. Moreover, for any time $t \in\left(-\theta_{2}, 0\right)$ and $\varepsilon>0$, we choose $\alpha \in W_{0}^{1, \infty}\left(-\theta_{1}, 0\right)$ with $\alpha(s)=\frac{\theta_{1}+s}{\theta_{1}-\theta_{2}}$ for $s \in\left(-\theta_{1},-\theta_{2}\right), \alpha \equiv 1$ on $\left(-\theta_{2}, t-\varepsilon\right), \alpha(s)=\frac{t-s}{\varepsilon}$ for $s \in(t-\varepsilon, t)$ and $\alpha \equiv 0$ on $(t, 0)$. Using the preceding estimate with this choice of cut-off functions and letting $\varepsilon \downarrow 0$, we infer

$$
\begin{aligned}
& \frac{1}{m} \int_{B_{\varrho_{2}} \times\{t\}} \int_{0}^{\left[u^{m}-k^{m}\right]_{+}}\left(k^{m}+\tau\right)^{\frac{1-m}{m}} \tau d \tau d x+\frac{1}{2} \int_{Q_{2}}\left|\nabla\left[u^{m}-k^{m}\right]_{+}\right|^{2} d z \\
& \leq \frac{8}{\left(\varrho_{1}-\varrho_{2}\right)^{2}} \int_{Q_{1}}\left[u^{m}-k^{m}\right]_{+}^{2} d z \\
&+\frac{1}{m\left(\theta_{1}-\theta_{2}\right)} \int_{Q_{1}} \int_{0}^{\left[u^{m}-k^{m}\right]_{+}}\left(k^{m}+\tau\right)^{\frac{1-m}{m}} \tau d \tau d z .
\end{aligned}
$$

This implies the claimed energy estimate by bounding the left-hand side from below via the inequality

$$
\frac{1}{m} \int_{0}^{\left[u^{m}-k^{m}\right]_{+}}\left(k^{m}+\tau\right)^{\frac{1-m}{m}} \tau d \tau \geq \frac{1}{m} u^{1-m} \int_{0}^{\left[u^{m}-k^{m}\right]_{+}} \tau d \tau=\frac{1}{2 m} u^{1-m}\left[u^{m}-k^{m}\right]_{+}^{2}
$$

and the right-hand side via

$$
\frac{1}{m} \int_{0}^{\left[u^{m}-k^{m}\right]_{+}}\left(k^{m}+\tau\right)^{\frac{1-m}{m}} \tau d \tau \leq \frac{k^{1-m}}{m} \int_{0}^{\left[u^{m}-k^{m}\right]_{+}} \tau d \tau=\frac{k^{1-m}}{2 m}\left[u^{m}-k^{m}\right]_{+}^{2} .
$$

Proof of (ii). Here, we consider an arbitrary $k>0$. Since most of the proof of (ii) is analogous to that of (i), we only indicate the necessary changes. As comparison maps, we now choose

$$
v_{h}^{m}:=\llbracket u^{m} \rrbracket_{h}+\left(\llbracket u^{m} \rrbracket_{h}-k^{m}\right)_{-}+\left\|\psi^{m}-\llbracket \psi^{m} \rrbracket_{h}\right\|_{L^{\infty}\left(\Omega_{T}\right)},
$$

which satisfy

$$
\partial_{t} v_{h}^{m}=\partial_{t} \max \left\{\llbracket u^{m} \rrbracket_{h}, k^{m}\right\} \in L^{\frac{m+1}{m}}\left(\Omega_{T}\right) .
$$

These maps are admissible since $v_{h}^{m} \geq \llbracket u^{m} \rrbracket_{h}+\left\|\psi^{m}-\llbracket \psi^{m} \rrbracket_{h}\right\|_{L^{\infty}\left(\Omega_{T}\right)} \geq \psi^{m}$ holds on $\Omega_{T}$. Now we can repeat the calculations leading to $(4.6)$, but now replacing $\left(\llbracket u^{m} \rrbracket_{h}-\right.$ $\left.k^{m}\right)_{+}$by $-\left(\llbracket u^{m} \rrbracket_{h}-k^{m}\right)_{-}$. Instead of the identity $(4.2)$, we now use

$$
\partial_{t} \llbracket u^{m} \rrbracket_{h}^{\frac{1}{m}}\left(-\left(\llbracket u^{m} \rrbracket_{h}-k^{m}\right)_{-}\right)=\frac{1}{m} \frac{\partial}{\partial t} \int_{0}^{\left(\llbracket u^{m} \rrbracket_{h}-k^{m}\right)_{-}}\left(k^{m}-\tau\right)^{\frac{1-m}{m}} \tau d \tau .
$$


Moreover, we replace (4.4) by $\nabla u^{m}=-\nabla\left[u^{m}-k^{m}\right]_{-}$on $\Omega_{T} \cap\{u \leq k\}$. The remainder of the proof works as in the case of (i), and analogously to (4.6), we derive the estimate

$$
\begin{aligned}
\frac{1}{m} \int_{B_{\varrho_{2}} \times\{t\}} \int_{0}^{\left[u^{m}-k^{m}\right]-}\left(k^{m}-\tau\right)^{\frac{1-m}{m}} & \tau d \tau d x+\frac{1}{2} \int_{Q_{2}}\left|\nabla\left[u^{m}-k^{m}\right]_{-}\right|^{2} d z \\
\leq & \frac{8}{\left(\varrho_{1}-\varrho_{2}\right)^{2}} \int_{Q_{1}}\left[u^{m}-k^{m}\right]_{-}^{2} d z \\
& +\frac{1}{m\left(\theta_{1}-\theta_{2}\right)} \int_{Q_{1}} \int_{0}^{\left[u^{m}-k^{m}\right]_{-}}\left(k^{m}-\tau\right)^{\frac{1-m}{m}} \tau d \tau d z
\end{aligned}
$$

for every $t \in\left(-\theta_{2}, 0\right)$. This implies the claim by estimating the left-hand side from below by

$$
\frac{1}{m} \int_{0}^{\left[u^{m}-k^{m}\right]_{-}}\left(k^{m}-\tau\right)^{\frac{1-m}{m}} \tau d \tau \geq \frac{k^{1-m}}{m} \int_{0}^{\left[u^{m}-k^{m}\right]_{-}} \tau d \tau=\frac{1}{2 m} k^{1-m}\left[u^{m}-k^{m}\right]_{-}^{2} .
$$

\subsection{The logarithmic estimate}

In this section we derive an estimate that will be useful later to compare the measures of certain super-level sets on different time slices. To this end, for parameters $0<\gamma<\Gamma$, we consider the function

$$
\phi(v):=\phi_{\Gamma, \gamma}(v):=\left[\log \left(\frac{\Gamma}{\Gamma+\gamma-v}\right)\right]_{+} \quad \text { for } v<\Gamma+\gamma .
$$

We note that $\phi(v)=0$ for $v \leq \gamma$, and for $v \leq \Gamma$ we have the estimates

$$
0 \leq \phi(v) \leq \log \left(\frac{\Gamma}{\gamma}\right) \quad \text { and } \quad 0 \leq \phi^{\prime}(v) \leq \frac{1}{\gamma} \quad \text { for } v \neq \gamma
$$

Moreover, the function satisfies the differential equation $\phi^{\prime \prime}=\left(\phi^{\prime}\right)^{2}$ for $v \neq \gamma$. We point out that contrary to $\phi$, the squared function $\phi^{2}$ is differentiable on $[0, \Gamma]$ with

$$
\left(\phi^{2}\right)^{\prime}=2 \phi \phi^{\prime} \text { on }[0, \Gamma] \text { and }\left(\phi^{2}\right)^{\prime \prime}=2(1+\phi)\left(\phi^{\prime}\right)^{2} \text { on }[0, \Gamma] \backslash\{\gamma\} \text {. }
$$

In particular $\left(\phi^{2}\right)^{\prime}$ is Lipschitz, which will be crucial in the proof below.

Lemma 4.2. We consider two concentric balls $B_{\varrho_{2}}\left(x_{o}\right) \Subset B_{\varrho_{1}}\left(x_{o}\right) \Subset \Omega$ and times $0<t_{1}<t_{2}<T$ and abbreviate $Q_{1}:=B_{\varrho_{1}}\left(x_{o}\right) \times\left(t_{1}, t_{2}\right)$. Let $u \in K_{\psi}\left(\Omega_{T}\right)$ be a locally bounded local weak solution to the obstacle problem for the porous medium equation in the sense of Definition 2.1 , for an obstacle $\psi \in C^{0}\left(\Omega_{T}, \mathbb{R}_{\geq 0}\right)$. For some $k>0$ 
with $k \geq \sup _{Q_{1}} \psi$ we define $\Gamma:=\sup _{Q_{1}}\left[u^{m}-k^{m}\right]_{+}$and denote by $\phi=\phi_{\Gamma, \gamma}$ the function introduced in (4.7) for some parameter $\gamma \in(0, \Gamma)$. Then we have

$$
\begin{aligned}
\sup _{t \in\left(t_{1}, t_{2}\right)} \int_{B_{\varrho_{2}}\left(x_{o}\right) \times\{t\}} u^{1-m} \phi^{2}\left(\left[u^{m}-k^{m}\right]_{+}\right) d x & \leq k^{1-m} \int_{B_{\varrho_{1}}\left(x_{o}\right) \times\left\{t_{1}\right\}} \phi^{2}\left(\left[u^{m}-k^{m}\right]_{+}\right) d x \\
& +\frac{8 m}{\left(\varrho_{1}-\varrho_{2}\right)^{2}} \int_{B_{\varrho_{1}}\left(x_{o}\right) \times\left(t_{1}, t_{2}\right)} \phi\left(\left[u^{m}-k^{m}\right]_{+}\right) d z .
\end{aligned}
$$

Proof. Since the asserted estimate is of local nature, we may assume $\psi \in$ $C^{0}\left(\overline{\Omega_{T}}\right)$. Because both sides of the asserted estimate are continuous in $k$, it suffices to prove the claim for every $k>\sup _{Q_{1}} \psi$. For the sake of convenience, we moreover assume $x_{o}=0$. In the variational inequality (2.2) we consider cut-off functions $\alpha \in W_{0}^{1, \infty}\left(\left(t_{1}, t_{2}\right), \mathbb{R}_{\geq 0}\right)$ and $\eta \in C_{0}^{1}\left(B_{\varrho_{1}}, \mathbb{R}_{\geq 0}\right)$. For a suitable $\lambda>0$, we wish to choose

$$
v_{h}^{m}:=\llbracket u^{m} \rrbracket_{h}-\lambda\left(\phi^{2}\right)^{\prime}\left(\left(\llbracket u^{m} \rrbracket_{h}-k^{m}\right)_{+}\right)+\left\|\psi^{m}-\llbracket \psi^{m} \rrbracket_{h}\right\|_{L^{\infty}}
$$

as comparison map in (2.2). Since $\left(\phi^{2}\right)^{\prime}$ is a Lipschitz map, the chain rule implies $\partial_{t} v_{h}^{m} \in L^{\frac{m+1}{m}}\left(\Omega_{T}\right)$ and $\nabla v_{h}^{m} \in L^{2}\left(\Omega_{T}\right)$. Moreover, $v_{h}$ satisfies the obstacle constraint for a sufficiently small choice of $\lambda>0$ since for $\llbracket u^{m} \rrbracket_{h} \leq k^{m}$ we have

$$
v_{h}^{m}=\llbracket u^{m} \rrbracket_{h}+\left\|\psi^{m}-\llbracket \psi^{m} \rrbracket_{h}\right\|_{L^{\infty}} \geq \psi^{m}
$$

by the obstacle condition for $u^{m}$, while for $\llbracket u^{m} \rrbracket_{h}>k^{m}$ we have

$$
v_{h}^{m}>k^{m}-\lambda\left(\phi^{2}\right)^{\prime}\left(\left(\llbracket u^{m} \rrbracket_{h}-k^{m}\right)_{+}\right) \geq \sup _{Q_{1}} \psi^{m},
$$

if we choose $\lambda \leq \frac{\left(k^{m}-\sup _{Q_{1}} \psi^{m}\right)}{\sup _{[0, \Gamma]}\left(\phi^{2}\right)^{\prime}}$. This means that $v_{h}^{m} \geq \psi^{m}$ holds on $Q_{1} \supset \operatorname{supp}(\alpha \eta)$, which makes $v_{h}$ admissible in (2.2). We thereby get

$$
\mathrm{I}_{h}+\mathrm{II}_{h}:=\left\langle\left\langle\partial_{t} u, \alpha \eta^{2}\left(v_{h}^{m}-u^{m}\right)\right\rangle\right\rangle+\int_{\Omega_{T}} \alpha \nabla u^{m} \cdot \nabla\left(\eta^{2}\left(v_{h}^{m}-u^{m}\right)\right) d z \geq 0 .
$$

For the analysis of $\mathrm{I}_{h}$, we first use Lemma 3.4 (i) to compute

$$
\begin{aligned}
\partial_{t} v_{h}^{m} & =\partial_{t} \llbracket u^{m} \rrbracket_{h}-\lambda\left(\phi^{2}\right)^{\prime \prime}\left(\left(\llbracket u^{m} \rrbracket_{h}-k^{m}\right)_{+}\right) \partial_{t} \llbracket u^{m} \rrbracket_{h} \\
& =\frac{1}{h}\left(u^{m}-\llbracket u^{m} \rrbracket_{h}\right)\left(1-\lambda\left(\phi^{2}\right)^{\prime \prime}\left(\left(\llbracket u^{m} \rrbracket_{h}-k^{m}\right)_{+}\right)\right) .
\end{aligned}
$$

We note that the terms involving $\left(\phi^{2}\right)^{\prime \prime}$ are a.e. well-defined since $\partial_{t} \llbracket u^{m} \rrbracket_{h}$ vanishes a.e. on the set $\left\{\llbracket u^{m} \rrbracket_{h}=k^{m}+\gamma\right\}$. By diminishing the value of $\lambda>0$ once more we can achieve $\lambda \leq\left[\sup _{[0, \Gamma]}\left(\phi^{2}\right)^{\prime \prime}\right]^{-1}$ and estimate

$$
\left(u-\llbracket u^{m} \rrbracket_{h}^{\frac{1}{m}}\right) \partial_{t} v_{h}^{m} \geq\left(u-\llbracket u^{m} \rrbracket_{h}^{\frac{1}{m}}\right) \frac{1}{h}\left(u^{m}-\llbracket u^{m} \rrbracket_{h}\right)\left(1-\lambda \sup \left(\phi^{2}\right)^{\prime \prime}\right) \geq 0 .
$$


This implies

$$
\begin{aligned}
\int_{\Omega_{T}} \alpha \eta^{2} u \partial_{t} v_{h}^{m} d z \geq & \int_{\Omega_{T}} \alpha \eta^{2} \llbracket u^{m} \rrbracket_{h}^{\frac{1}{m}} \partial_{t} v_{h}^{m} d z \\
= & \int_{\Omega_{T}} \alpha \eta^{2} \llbracket u^{m} \rrbracket_{h}^{\frac{1}{m}} \partial_{t}\left(\llbracket u^{m} \rrbracket_{h}-\lambda\left(\phi^{2}\right)^{\prime}\left(\left(\llbracket u^{m} \rrbracket_{h}-k^{m}\right)_{+}\right)\right) d z \\
= & \int_{\Omega_{T}} \eta^{2}\left(\alpha \frac{m}{m+1} \partial_{t} \llbracket u^{m} \rrbracket_{h}^{\frac{m+1}{m}}+\lambda \alpha^{\prime} \llbracket u^{m} \rrbracket_{h}^{\frac{1}{m}}\left(\phi^{2}\right)^{\prime}\left(\left(\llbracket u^{m} \rrbracket_{h}-k^{m}\right)_{+}\right)\right) d z \\
& +\lambda \int_{\Omega_{T}} \alpha \eta^{2} \partial_{t} \llbracket u^{m} \rrbracket_{h}^{\frac{1}{m}}\left(\phi^{2}\right)^{\prime}\left(\left(\llbracket u^{m} \rrbracket_{h}-k^{m}\right)_{+}\right) d z .
\end{aligned}
$$

We re-write the last integrand using the equation

$$
\partial_{t} \llbracket u^{m} \rrbracket_{h}^{\frac{1}{m}}\left(\phi^{2}\right)^{\prime}\left(\left(\llbracket u^{m} \rrbracket_{h}-k^{m}\right)_{+}\right)=\frac{1}{m} \frac{\partial}{\partial t} \int_{0}^{\left(\llbracket u^{m} \rrbracket_{h}-k^{m}\right)_{+}}\left(k^{m}+\tau\right)^{\frac{1-m}{m}}\left(\phi^{2}\right)^{\prime}(\tau) d \tau .
$$

Combining the two preceding formulae and integrating by parts we deduce

$$
\begin{aligned}
\int_{\Omega_{T}} \alpha \eta^{2} u \partial_{t} v_{h}^{m} d z \geq & \int_{\Omega_{T}} \eta^{2} \alpha^{\prime}\left(-\frac{m}{m+1} \llbracket u^{m} \rrbracket_{h}^{\frac{m+1}{m}}+\lambda \llbracket u^{m} \rrbracket_{h}^{\frac{1}{m}}\left(\phi^{2}\right)^{\prime}\left(\left(\llbracket u^{m} \rrbracket_{h}-k^{m}\right)_{+}\right)\right) d z \\
& -\frac{\lambda}{m} \int_{\Omega_{T}} \alpha^{\prime} \eta^{2} \int_{0}^{\left(\llbracket u^{m} \rrbracket_{h}-k^{m}\right)_{+}}\left(k^{m}+\tau\right)^{\frac{1-m}{m}}\left(\phi^{2}\right)^{\prime}(\tau) d \tau d z .
\end{aligned}
$$

Now we recall the definition (2.1) to conclude

$$
\begin{aligned}
\mathrm{I}_{h}= & \int_{\Omega_{T}} \eta^{2}\left[\alpha^{\prime}\left[\frac{1}{m+1} u^{m+1}-u v_{h}^{m}\right]-\alpha u \partial_{t} v_{h}^{m}\right] d z \\
\leq & \int_{\Omega_{T}} \eta^{2} \alpha^{\prime}\left[\frac{1}{m+1} u^{m+1}-u\left(\llbracket u^{m} \rrbracket_{h}-\lambda\left(\phi^{2}\right)^{\prime}\left(\left(\llbracket u^{m} \rrbracket_{h}-k^{m}\right)_{+}\right)+\left\|\psi^{m}-\llbracket \psi^{m} \rrbracket_{h}\right\|_{L^{\infty}}\right)\right] d z \\
& -\int_{\Omega_{T}} \eta^{2} \alpha^{\prime}\left(-\frac{m}{m+1} \llbracket u^{m} \rrbracket_{h}^{\frac{m+1}{m}}+\lambda \llbracket u^{m} \rrbracket_{h}^{\frac{1}{m}}\left(\phi^{2}\right)^{\prime}\left(\left(\llbracket u^{m} \rrbracket_{h}-k^{m}\right)_{+}\right)\right) d z \\
& +\frac{\lambda}{m} \int_{\Omega_{T}} \alpha^{\prime} \eta^{2} \int_{0}^{\left(\llbracket u^{m} \rrbracket_{h}-k^{m}\right)_{+}}\left(k^{m}+\tau\right)^{\frac{1-m}{m}}\left(\phi^{2}\right)^{\prime}(\tau) d \tau d z .
\end{aligned}
$$

Because $\llbracket u^{m} \rrbracket_{h} \rightarrow u^{m}$ in $L^{\frac{m+1}{m}}\left(Q_{1}\right)$ and $\left\|\psi^{m}-\llbracket \psi^{m} \rrbracket_{h}\right\|_{L^{\infty}} \rightarrow 0$ as $h \downarrow 0$ by Lemma 3.4, the first two integrals on the right-hand side cancel each other in the limit $h \downarrow 0$. Letting $h \downarrow 0$ therefore yields

$$
\limsup _{h \downarrow 0} \mathrm{I}_{h} \leq \frac{\lambda}{m} \int_{\Omega_{T}} \alpha^{\prime} \eta^{2} \int_{0}^{\left[u^{m}-k^{m}\right]_{+}}\left(k^{m}+\tau\right)^{\frac{1-m}{m}}\left(\phi^{2}\right)^{\prime}(\tau) d \tau d z .
$$

Now, we turn our attention to the term $\mathrm{II}_{h}$. We claim that

$$
\eta^{2}\left(v_{h}^{m}-u^{m}\right) \rightarrow-\lambda \eta^{2}\left(\phi^{2}\right)^{\prime}\left(\left[u^{m}-k^{m}\right]_{+}\right) \quad \text { weakly in } L^{2}\left(t_{1}, t_{2} ; H^{1}\left(B_{\varrho_{1}}\right)\right),
$$


as $h \downarrow 0$. First we note that this convergence holds strongly in $L^{2}\left(Q_{1}\right)$ by Lemma 3.4 (i) and (iii). Furthermore, the sequence on the left-hand side is bounded in $L^{2}\left(t_{1}, t_{2} ; H^{1}\left(B_{\varrho_{1}}\right)\right)$ by Lemma 3.4 (ii) and because $\left(\phi^{2}\right)^{\prime}$ is Lipschitz. This implies the claimed weak convergence, which in turn implies

$$
\begin{aligned}
\lim _{h \downarrow 0} \mathrm{II}_{h}= & -\lambda \int_{\Omega_{T}} \alpha \nabla u^{m} \cdot \nabla\left[\eta^{2}\left(\phi^{2}\right)^{\prime}\left(\left[u^{m}-k^{m}\right]_{+}\right)\right] d z \\
= & -\lambda \int_{\Omega_{T}} \alpha \eta^{2}\left|\nabla u^{m}\right|^{2}\left(\phi^{2}\right)^{\prime \prime}\left(\left[u^{m}-k^{m}\right]_{+}\right) d z \\
& +2 \lambda \int_{\Omega_{T}} \alpha \eta \nabla u^{m} \cdot \nabla \eta\left(\phi^{2}\right)^{\prime}\left(\left[u^{m}-k^{m}\right]_{+}\right) d z .
\end{aligned}
$$

The last integral is well-defined since $\nabla u^{m}=0$ a.e. on the set $\left\{u^{m}=k^{m}+\gamma\right\}$. Next, we apply Young's inequality in the last integral and infer

$$
\begin{aligned}
\lim _{h \downarrow 0} \mathrm{II}_{h} \leq & \lambda \int_{\Omega_{T}} \alpha \eta^{2}\left|\nabla u^{m}\right|^{2}\left[2 \phi\left(\phi^{\prime}\right)^{2}-\left(\phi^{2}\right)^{\prime \prime}\right]\left(\left[u^{m}-k^{m}\right]_{+}\right) d z \\
& +2 \lambda \int_{\Omega_{T}} \alpha|\nabla \eta|^{2} \phi\left(\left[u^{m}-k^{m}\right]_{+}\right) d z \\
\leq & 2 \lambda \int_{\Omega_{T}} \alpha|\nabla \eta|^{2} \phi\left(\left[u^{m}-k^{m}\right]_{+}\right) d z .
\end{aligned}
$$

The last estimate is a consequence of (4.9), which implies $2 \phi\left(\phi^{\prime}\right)^{2}-\left(\phi^{2}\right)^{\prime \prime}=$ $-2\left(\phi^{\prime}\right)^{2} \leq 0$. Next, we plug (4.11) and (4.12) into (4.10) and divide by $\lambda$. This provides us with the estimate

$$
\begin{aligned}
\frac{1}{m} \int_{\Omega_{T}} \alpha^{\prime} \eta^{2} \int_{0}^{\left[u^{m}-k^{m}\right]_{+}} & \left(k^{m}+\tau\right)^{\frac{1-m}{m}}\left(\phi^{2}\right)^{\prime}(\tau) d \tau d z \\
& +2 \int_{\Omega_{T}} \alpha|\nabla \eta|^{2} \phi\left(\left[u^{m}-k^{m}\right]_{+}\right) d z \geq 0 .
\end{aligned}
$$

Now we choose $\eta \in C_{0}^{1}\left(B_{\varrho_{1}}, \mathbb{R}_{\geq 0}\right)$ as a standard cut-off function with $\eta \equiv 1$ on $B_{\varrho_{2}}$ and $|\nabla \eta| \leq \frac{2}{\varrho_{1}-\varrho_{2}}$ on $B_{\varrho_{1}}$. For the choice of $\alpha$, we fix $t \in\left(t_{1}, t_{2}\right)$ and some $0<\varepsilon<\frac{1}{2}\left(t-t_{1}\right)$. Then we define $\alpha \in W_{0}^{1, \infty}\left(\left(t_{1}, t_{2}\right), \mathbb{R}_{\geq 0}\right)$ by $\alpha(s)=\frac{s-t_{1}}{\varepsilon}$ for $s \in\left(t_{1}, t_{1}+\varepsilon\right), \alpha \equiv 1$ on $\left(t_{1}+\varepsilon, t-\varepsilon\right), \alpha(s)=\frac{t-s}{\varepsilon}$ for $s \in(t-\varepsilon, t)$ and $\alpha \equiv 0$ elsewhere. Exploiting the preceding inequality with this choice of cut-off functions and letting $\varepsilon \downarrow 0$, we deduce

$$
\begin{aligned}
\frac{1}{m} \int_{B_{\varrho_{2}} \times\{t\}} & \int_{0}^{\left[u^{m}-k^{m}\right]_{+}}\left(k^{m}+\tau\right)^{\frac{1-m}{m}}\left(\phi^{2}\right)^{\prime}(\tau) d \tau d x \\
\leq & \frac{1}{m} \int_{B_{\varrho_{1}} \times\left\{t_{1}\right\}} \int_{0}^{\left[u^{m}-k^{m}\right]_{+}}\left(k^{m}+\tau\right)^{\frac{1-m}{m}}\left(\phi^{2}\right)^{\prime}(\tau) d \tau d x \\
& +\frac{8}{\left(\varrho_{1}-\varrho_{2}\right)^{2}} \int_{Q_{1}} \phi\left(\left[u^{m}-k^{m}\right]_{+}\right) d z
\end{aligned}
$$


For the estimate of the first two integrals, we use the inequalities

$$
u^{1-m} \leq\left(k^{m}+\tau\right)^{\frac{1-m}{m}} \leq k^{1-m} \quad \text { if } 0<\tau<\left[u^{m}-k^{m}\right]_{+}
$$

and arrive at the claimed estimate.

\section{Local boundedness of solutions}

Theorem 5.1. We consider an obstacle $\psi \in C^{0}\left(\Omega_{T}, \mathbb{R}_{\geq 0}\right)$. Then every local weak solution $u \in K_{\psi}\left(\Omega_{T}\right)$ of the obstacle problem to the porous medium equation in the sense of Definition 2.1 satisfies $u \in L_{\mathrm{loc}}^{\infty}\left(\Omega_{T}\right)$ and we have the local estimate

$$
\sup _{Q_{\varrho, \theta}\left(z_{o}\right)} u \leq c \max \left\{\left(\frac{1}{\varrho^{n+2}} \int_{Q_{2 \varrho, 2 \theta}\left(z_{o}\right)} u^{2 m} d z\right)^{\frac{1}{m+1}}, \sup _{Q_{2 \varrho, 2 \theta}\left(z_{o}\right)} \psi,\left(\frac{\varrho^{2}}{\theta}\right)^{\frac{1}{m-1}}\right\},
$$

for every parabolic cylinder $Q_{2 \varrho, 2 \theta}\left(z_{o}\right) \Subset \Omega_{T}$, with a constant $c=c(n, m)$.

Proof. For any $i \in \mathbb{N}_{0}$, we define

$$
\varrho_{i}:=\varrho+\frac{1}{2^{i}} \varrho \quad \text { and } \quad \theta_{i}:=\theta+\frac{1}{2^{i}} \theta
$$

and abbreviate $Q_{i}:=Q_{\varrho_{i}, \theta_{i}}\left(z_{o}\right)$. For notational convenience we assume $z_{o}=0$ throughout the proof. We define $k>0$ by

$$
k^{m}:=\max \left\{\left(\frac{c_{o}}{\varrho^{n+2}} \int_{Q_{0}} u^{2 m} d z\right)^{\frac{m}{m+1}}, 4 \sup _{Q_{0}} \psi^{m},\left(\frac{\varrho^{2}}{\theta}\right)^{\frac{m}{m-1}}\right\},
$$

for a constant $c_{o}>0$ to be chosen large on later in dependence on $n$ and $m$ only. For this choice of $k$, we introduce an increasing sequence of levels $k_{i}$ and intermediate levels $\tilde{k}_{i}$ by

$$
k_{i}^{m}:=k^{m}-\frac{1}{2^{i}} k^{m} \quad \text { and } \quad \tilde{k}_{i}^{m}:=\frac{1}{2}\left(k_{i}^{m}+k_{i+1}^{m}\right)=k^{m}-\frac{3}{2^{i+2}} k^{m}
$$

for $i \in \mathbb{N}_{0}$. For any $i \in \mathbb{N}_{0}$, we apply Lemma 4.1 (i) on the cylinders $Q_{i+1} \subset Q_{i} \subset \Omega_{T}$ and the level $\tilde{k}_{i}^{m} \geq \frac{1}{4} k^{m} \geq \sup _{Q_{i}} \psi^{m}$, which provides us with the estimate

$$
\begin{array}{rl}
\sup _{t \in\left(-\theta_{i+1}, 0\right)} \int_{B_{\varrho_{i+1}} \times\{t\}} u^{1-m}\left[u^{m}-\tilde{k}_{i}^{m}\right]_{+}^{2} & d x+\int_{Q_{i+1}}\left|\nabla\left[u^{m}-\tilde{k}_{i}^{m}\right]_{+}\right|^{2} d z \\
\leq c\left(\frac{1}{\left(\varrho_{i}-\varrho_{i+1}\right)^{2}}+\frac{k^{1-m}}{\theta_{i}-\theta_{i+1}}\right) \int_{Q_{i}}\left[u^{m}-\tilde{k}_{i}^{m}\right]_{+}^{2} d z
\end{array}
$$


Here and in the remainder of the proof, we write $c$ for universal constants that depend at most on $n$ and $m$. Using moreover the facts $k^{1-m} \leq \frac{\theta}{\varrho^{2}}$ and $k_{i}<\tilde{k}_{i}<k_{i+1}$, we deduce

$$
\begin{array}{r}
\sup _{t \in\left(-\theta_{i+1}, 0\right)} \int_{B_{\varrho_{i+1}} \times\{t\}} u^{1-m}\left[u^{m}-\tilde{k}_{i}^{m}\right]_{+}^{2} d x+\int_{Q_{i+1}}\left|\nabla\left[u^{m}-k_{i+1}^{m}\right]_{+}\right|^{2} d z \\
\leq \frac{c 2^{2 i}}{\varrho^{2}} \int_{Q_{i}}\left[u^{m}-k_{i}^{m}\right]_{+}^{2} d z .
\end{array}
$$

In order to bound the first integral on the left-hand side from below, we obtain from a straightforward calculation

$$
u^{m} \leq \frac{k_{i+1}^{m}}{k_{i+1}^{m}-\tilde{k}_{i}^{m}}\left(u^{m}-\tilde{k}_{i}^{m}\right)=\left(2^{i+2}-2\right)\left[u^{m}-\tilde{k}_{i}^{m}\right]_{+}, \quad \text { provided } u \geq k_{i+1},
$$

and consequently,

$$
\left[u^{m}-k_{i+1}^{m}\right]_{+}^{1+\frac{1}{m}} \leq 2^{i+2} u^{1-m}\left[u^{m}-\tilde{k}_{i}^{m}\right]_{+}^{2} \quad \text { a.e. on } \Omega_{T} \text {. }
$$

Using this estimate in (5.2), we deduce

$$
\begin{array}{r}
\sup _{t \in\left(-\theta_{i+1}, 0\right)} \int_{B_{\varrho_{i+1}} \times\{t\}}\left[u^{m}-k_{i+1}^{m}\right]_{+}^{1+\frac{1}{m}} d x+\int_{Q_{i+1}}\left|\nabla\left[u^{m}-k_{i+1}^{m}\right]_{+}\right|^{2} d z \\
\leq \frac{c 2^{3 i}}{\varrho^{2}} \int_{Q_{i}}\left[u^{m}-k_{i}^{m}\right]_{+}^{2} d z
\end{array}
$$

for all $i \in \mathbb{N}_{0}$. Next, we apply the Gagliardo-Nirenberg inequality from Lemma 3.1 with the choices $p=2, r=1+\frac{1}{m}$ and $q=\frac{2}{m n}(m n+m+1)>2$ to the function $\left[u^{m}-\right.$ $\left.k_{i+1}^{m}\right]_{+}$on $Q_{i+1}$, with the result

$$
\begin{aligned}
\int_{Q_{i+1}}\left[u^{m}-k_{i+1}^{m}\right]_{+}^{q} d z \leq & c\left(\sup _{t \in\left(-\theta_{i+1}, 0\right)} \int_{B_{\varrho_{i+1}} \times\{t\}}\left[u^{m}-k_{i+1}^{m}\right]_{+}^{1+\frac{1}{m}} d x\right)^{\frac{2}{n}} \\
& \times \int_{Q_{i+1}}\left(\left|\nabla\left[u^{m}-k_{i+1}^{m}\right]_{+}\right|^{2}+\left|\frac{\left[u^{m}-k_{i+1}^{m}\right]_{+}}{\varrho}\right|^{2}\right) d z .
\end{aligned}
$$

We estimate the right-hand side by means of (5.3) and arrive at

$$
\int_{Q_{i+1}}\left[u^{m}-k_{i+1}^{m}\right]_{+}^{q} d z \leq c\left(\frac{2^{3 i}}{\varrho^{2}} \int_{Q_{i}}\left[u^{m}-k_{i}^{m}\right]_{+}^{2} d z\right)^{1+\frac{2}{n}} .
$$


Now we first use Hölder's inequality and then (5.4), with the result

$$
\begin{array}{rl}
\int_{Q_{i+1}}\left[u^{m}-k_{i+1}^{m}\right]_{+}^{2} & d z \\
\leq\left|Q_{i+1} \cap\left\{u \geq k_{i+1}\right\}\right|^{1-\frac{2}{q}}\left(\int_{Q_{i+1}}\left[u^{m}-k_{i+1}^{m}\right]_{+}^{q} d z\right)^{\frac{2}{q}} \\
\leq\left(\frac{1}{\left(k_{i+1}^{m}-k_{i}^{m}\right)^{2}} \int_{Q_{i+1}}\left[u^{m}-k_{i}^{m}\right]_{+}^{2} d z\right)^{1-\frac{2}{q}}\left(\int_{Q_{i+1}}\left[u^{m}-k_{i+1}^{m}\right]_{+}^{q} d z\right)^{\frac{2}{q}} \\
\leq \frac{c 2^{\left(2+\frac{2}{q}+\frac{12}{q n}\right) i}}{k^{2 m\left(1-\frac{2}{q}\right)} \varrho^{\left(1+\frac{2}{n}\right) \frac{4}{q}}}\left(\int_{Q_{i}}\left[u^{m}-k_{i}^{m}\right]_{+}^{2} d z\right)^{1+\frac{4}{q n}} .
\end{array}
$$

For the sequence of integrals $Y_{i}:=\int_{Q_{i}}\left[u^{m}-k_{i}^{m}\right]_{+}^{2} d z$ for $i \in \mathbb{N}_{0}$, we therefore have established the estimate

$$
Y_{i+1} \leq C B^{i} Y_{i}^{1+\frac{4}{q n}} \quad \text { for all } i \in \mathbb{N}_{0},
$$

with

$$
C=\left(\frac{c}{k^{m+1} \varrho^{n+2}}\right)^{\frac{4}{q n}} \text { and } B=64 .
$$

From the choice of $k$ in $(5.1)$ we infer

$$
k^{m+1} \geq \frac{c_{o}}{\varrho^{n+2}} \int_{Q_{0}} u^{2 m} d z=\frac{c_{o}}{\varrho^{n+2}} Y_{0} .
$$

For the parameter $\alpha:=\frac{4}{q n}$, this implies

$$
C^{-\frac{1}{\alpha}} B^{-\frac{1}{\alpha^{2}}}=c^{-1} k^{m+1} \varrho^{n+2} B^{-\frac{q^{2} n^{2}}{16}} \geq c^{-1} c_{o} B^{-\frac{q^{2} n^{2}}{16}} Y_{0}
$$

At this stage, we choose $c_{o}:=c B^{\frac{q^{2} n^{2}}{16}}$. This choice fixes the constant in dependence of $m$ and $n$ and yields the bound

$$
Y_{0} \leq C^{-\frac{1}{\alpha}} B^{-\frac{1}{\alpha^{2}}}
$$

Because of (5.5) and (5.6), the assumptions of Lemma 3.2 are satisfied for $\alpha=\frac{4 q}{n}$. Hence, we infer

$$
0=\lim _{i \rightarrow \infty} Y_{i}=\int_{Q_{\varrho}, \theta}\left[u^{m}-k^{m}\right]_{+}^{2} d z,
$$

which is equivalent to $u^{m} \leq k^{m}$ a.e. on $Q_{\varrho, \theta}$. In view of the choice of $k$ in (5.1), this completes the proof of the theorem. 


\section{Hölder continuity}

In this section we will prove the assertion of Theorem 1.1 that solutions to the obstacle problem for the porous medium equation are Hölder continuous, provided that the obstacle function is Hölder continuous. Therefore, we may assume throughout this section that there exists $\beta \in(0,1)$ such that

$$
\psi^{m} \in C^{0 ; \beta, \beta / 2}\left(\Omega_{T}\right) .
$$

By $C^{0 ; \beta, \beta / 2}$ we mean the space of functions which are Hölder continuous with Hölder exponent $\beta$ in space and $\beta / 2$ in time. More precisely, for a function $f: \Omega_{T} \rightarrow \mathbb{R}$ we define

$$
[f]_{0 ; \beta, \beta / 2}:=\sup _{(x, t),(y, s) \in \Omega_{T}} \frac{|f(x, t)-f(y, s)|}{\max \left\{|x-y|^{\beta},|t-s|^{\beta / 2}\right\}} .
$$

Then, $f \in C^{0 ; \beta, \beta / 2}\left(\Omega_{T}\right)$ if and only if $[f]_{0 ; \beta, \beta / 2}<\infty$.

Hölder continuity at a point follows by constructing a sequence of cylinders shrinking to the point. Each of the cylinders should be roughly half the size of the previous one, and the oscillation of the function should be reduced by a fixed multiplicative factor when passing to the next cylinder. We also need to ensure that the cylinders have a proper scaling to balance the different powers in the energy estimates.

\subsection{Two alternatives}

The oscillation can be reduced by either increasing the infimum or decreasing the supremum of a function. Thus Hölder continuity will follow from Lemmas 6.1 and 6.2 below. We call Lemma 6.1 the first alternative, and Lemma 6.2 the second alternative, since for a given cylinder, either (6.2) holds, or (6.4) holds with $\nu=\nu_{0}$.

To introduce the proper scaling, throughout this section we fix parameters $0 \leq \mu_{-} \leq \mu_{+}$and $\theta \geq 0$ and define

$$
\omega^{m}:=\mu_{+}^{m}-\mu_{-}^{m} .
$$

It will be necessary to distinguish between cylinders where the oscillation is large compared to the infimum of $u$, (note that (D) below implies that $\mu_{-} \leq \omega$ ) and cylinders where the oscillation is small compared to the infimum of $u$. Thus we assume throughout this subsection that either

$$
\mu_{-} \leq \frac{1}{2} \mu_{+} \quad \text { and } \quad \theta=\omega^{1-m}
$$

(which we call the degenerate regime) or

$$
\mu_{-}>\frac{1}{2} \mu_{+} \quad \text { and } \quad\left(2 \mu_{+}\right)^{1-m} \leq \theta \leq\left(\frac{1}{2} \mu_{+}\right)^{1-m}
$$

(which we call the nondegenerate regime) holds true. 
Lemma 6.1. Suppose that $Q_{2 \varrho, \theta(2 \varrho)^{2}}\left(z_{o}\right) \subset \Omega_{T}$ is a parabolic cylinder satisfying

$$
\inf _{Q_{2 \varrho, \theta(2 \varrho)^{2}}\left(z_{o}\right)} u \geq \mu_{-}, \quad \text { and } \quad \sup _{Q_{2 \varrho, \theta(2 \varrho)^{2}}\left(z_{o}\right)} u \leq \mu_{+} .
$$

Then there exists a number $\nu_{o}=\nu_{o}(n, m) \in(0,1)$, such that if

$$
\left|Q_{\varrho, \theta \varrho^{2}}\left(z_{o}\right) \cap\left\{u^{m} \leq \mu_{-}^{m}+\frac{1}{2} \omega^{m}\right\}\right| \leq \nu_{o}\left|Q_{\varrho, \theta \varrho^{2}}\right|,
$$

then

$$
u^{m} \geq \mu_{-}^{m}+\frac{1}{4} \omega^{m} \quad \text { a.e. in } Q_{\varrho / 2, \theta(\varrho / 2)^{2}}\left(z_{o}\right) \text {. }
$$

Lemma 6.2. Suppose that $Q_{2 \varrho, \theta(2 \varrho)^{2}}\left(z_{o}\right) \subset \Omega_{T}$ is a parabolic cylinder satisfying

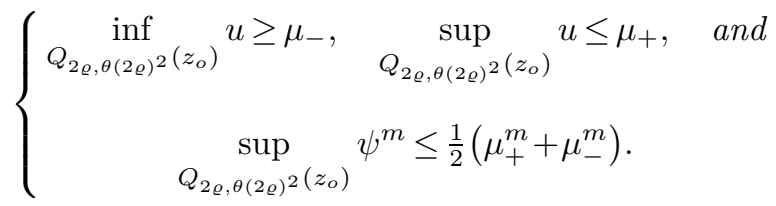

Then, for any $\nu \in(0,1)$ there exists a constant $a=a(n, m, \nu) \in\left(0, \frac{1}{4}\right]$ such that if

$$
\left|Q_{\varrho, \theta \varrho^{2}}\left(z_{o}\right) \cap\left\{u^{m} \leq \mu_{-}^{m}+\frac{1}{2} \omega^{m}\right\}\right|>\nu\left|Q_{\varrho, \theta \varrho^{2}}\right|,
$$

then

$$
u^{m} \leq \mu_{+}^{m}-a \omega^{m} \quad \text { a.e. in } Q_{\varrho / 2, \frac{1}{2} \nu \theta(\varrho / 2)^{2}}\left(z_{o}\right) .
$$

\subsubsection{The first alternative}

Proof of Lemma 6.1. For $i \in \mathbb{N}_{0}$ we define

$$
\left(\xi_{i}\right)^{m}:=\frac{1}{4}+\frac{1}{2^{i+2}}, \quad k_{i}^{m}:=\mu_{-}^{m}+\left(\xi_{i} \omega\right)^{m}, \quad \text { and } \quad \varrho_{i}:=\frac{\varrho}{2}+\frac{\varrho}{2^{i+1}} .
$$

Then, we have that $\left(\xi_{o}\right)^{m}=\frac{1}{2}, \xi_{i}$ is decreasing and $\left(\xi_{i}\right)^{m} \downarrow \frac{1}{4}$ as $i \rightarrow \infty$. Similarly, we have that $\varrho_{o}=\varrho, \varrho_{i}$ is decreasing and $\varrho_{i} \downarrow \frac{1}{2} \varrho$ as $i \rightarrow \infty$. Moreover, we abbreviate

$$
Q_{i}:=Q_{\varrho_{i}, \theta \varrho_{i}^{2}}\left(z_{o}\right)
$$

and

$$
Y_{i}:=\frac{\left|Q_{i} \cap\left\{u<k_{i}\right\}\right|}{\left|Q_{i}\right|} .
$$

We aim at deriving an estimate for $Y_{i+1}$ in terms of $Y_{i}$ so that fast geometric convergence from Lemma 3.2 can be applied. We use the fact that

$$
\left[u^{m}-k_{i}^{m}\right]_{-} \geq k_{i}^{m}-k_{i+1}^{m}=\frac{\omega^{m}}{2^{i+3}} \quad \text { in the set }\left\{u<k_{i+1}\right\}
$$


to get

$$
\begin{aligned}
\int_{Q_{i+1}}\left[u^{m}-k_{i}^{m}\right]_{-}^{\frac{2(n+2)}{n}} d z & \geq \int_{Q_{i+1} \cap\left\{u<k_{i+1}\right\}}\left[u^{m}-k_{i}^{m}\right]_{-}^{\frac{2(n+2)}{n}} d z \\
& \geq \frac{\omega^{\frac{2 m(n+2)}{n}}}{8^{\frac{2(n+2)}{n}} 2^{\frac{2 i(n+2)}{n}}}\left|Q_{i+1} \cap\left\{u<k_{i+1}\right\}\right| .
\end{aligned}
$$

Next, we apply Gagliardo-Nirenberg's inequality from Lemma 3.1 and the energy estimate from Lemma 4.1 (ii) to get

$$
\begin{aligned}
\int_{Q_{i+1}}\left[u^{m}-k_{i}^{m}\right]_{-}^{\frac{2(n+2)}{n}} d z & \left.\sup _{t \in\left(t_{o}-\theta \varrho_{i+1}^{2}, t_{o}\right)} \int_{B_{\varrho_{i+1}}}\left[u^{m}-k_{i}^{m}\right]_{-}^{2} d x\right)^{\frac{2}{n}} \\
& \times \int_{Q_{i+1}}\left(\left|\nabla\left[u^{m}-k^{m}\right]_{-}\right|^{2}+\left|\frac{\left[u^{m}-k_{i}^{m}\right]_{-}}{\varrho_{i+1}}\right|^{2}\right) d z \\
\leq & \frac{c 2^{\frac{2 i(n+2)}{n}} k_{i}^{\frac{2(m-1)}{n}}}{\varrho^{\frac{2(n+2)}{n}}} \\
& \times\left(\int_{Q_{i}}\left[u^{m}-k_{i}^{m}\right]_{-}^{2} d z+\frac{1}{\theta} \int_{Q_{i}} \int_{0}^{\left[u^{m}-k_{i}^{m}\right]_{-}}\left(k_{i}^{m}-\tau\right)^{\frac{1-m}{m}} \tau d \tau d z\right)^{1+\frac{2}{n}},
\end{aligned}
$$

where $c=c(n, m)$. If $(\mathrm{D})$ is satisfied, we estimate

$$
\begin{aligned}
\int_{0}^{\left[u^{m}-k_{i}^{m}\right]_{-}}\left(k_{i}^{m}-\tau\right)^{\frac{1-m}{m}} \tau d \tau & \leq\left[u^{m}-k_{i}^{m}\right]_{-} \int_{0}^{\left[u^{m}-k_{i}^{m}\right]_{-}}\left(k_{i}^{m}-\tau\right)^{\frac{1-m}{m}} d \tau \\
& =m\left[u^{m}-k_{i}^{m}\right]_{-}\left[u-k_{i}\right]_{-} \leq m\left[u^{m}-k_{i}^{m}\right]_{-}^{1+\frac{1}{m}} .
\end{aligned}
$$

Now, we use the fact that

$$
\left[u^{m}-k_{i}^{m}\right]_{-} \leq k_{i}^{m}-\mu_{-}^{m}=\left(\xi_{i} \omega\right)^{m} \leq \omega^{m},
$$

which holds due to the assumption $\mu_{-} \leq \inf _{Q_{0}} u$, and since $\theta=\omega^{1-m}$, we obtain

$$
\begin{aligned}
\int_{Q_{i+1}}\left[u^{m}-k_{i}^{m}\right]_{-}^{\frac{2(n+2)}{n}} & d z \\
& \leq \frac{c 2^{\frac{2 i(n+2)}{n} k_{i}^{\frac{2(m-1)}{n}}}\left(\omega^{2 m}+\frac{\omega^{m+1}}{\theta}\right)^{1+\frac{2}{n}}\left|Q_{i} \cap\left\{u<k_{i}\right\}\right|^{1+\frac{2}{n}}}{\varrho^{\frac{2(n+2)}{n}}} \\
& \leq \frac{c 2^{\frac{2 i(n+2)}{n}} k_{i}^{\frac{2(m-1)}{n}} \omega^{\frac{2 m(n+2)}{n}}}{\varrho^{\frac{2(n+2)}{n}}}\left|Q_{i} \cap\left\{u<k_{i}\right\}\right|^{1+\frac{2}{n}} .
\end{aligned}
$$


Otherwise, if $(\mathrm{N})$ is satisfied, we observe that $\inf _{Q_{0}} u \geq \mu_{-}>0$ and estimate

$$
\int_{0}^{\left[u^{m}-k_{i}^{m}\right]_{-}}\left(k_{i}^{m}-\tau\right)^{\frac{1-m}{m}} \tau d \tau \leq u^{1-m} \int_{0}^{\left[u^{m}-k_{i}^{m}\right]_{-}} \tau d \tau=\frac{1}{2} u^{1-m}\left[u^{m}-k_{i}^{m}\right]_{-}^{2} .
$$

Using again the fact that $\left[u^{m}-k_{i}^{m}\right]_{-} \leq \omega^{m}$ we get

$$
\int_{Q_{i+1}}\left[u^{m}-k_{i}^{m}\right]_{-}^{\frac{2(n+2)}{n}} d z \leq \frac{c 2^{\frac{2 i(n+2)}{n} k_{i}^{\frac{2(m-1)}{n}}}}{\varrho^{\frac{2(n+2)}{n}}}\left(\omega^{2 m}+\frac{\mu_{-}^{1-m} \omega^{2 m}}{\theta}\right)^{1+\frac{2}{n}}\left|Q_{i} \cap\left\{u<k_{i}\right\}\right|^{1+\frac{2}{n}} .
$$

Using $\mu_{-}^{1-m}<\left(\frac{1}{2} \mu_{+}\right)^{1-m} \leq 4^{m-1} \theta$, we obtain (6.6) also in the case that (N) is satisfied. Combining (6.6) with (6.5), we get

$$
\left|Q_{i+1} \cap\left\{u<k_{i+1}\right\}\right| \leq \frac{c 4^{\frac{2 i(n+2)}{n}} k_{i}^{\frac{2(m-1)}{n}}}{\varrho^{\frac{2(n+2)}{n}}}\left|Q_{i} \cap\left\{u<k_{i}\right\}\right|^{1+\frac{2}{n}}
$$

Dividing on both sides by $\left|Q_{i+1}\right|$ and recalling the definition of $Y_{i}$, we get

$$
Y_{i+1} \leq \frac{c b^{i} k_{i}^{\frac{2(m-1)}{n}}\left|Q_{i}\right|^{1+\frac{2}{n}}}{\varrho^{2\left(1+\frac{2}{n}\right)}\left|Q_{i+1}\right|} Y_{i}^{1+\frac{2}{n}}
$$

where we abbreviated $b:=4^{\frac{2(n+2)}{n}}$. If (D) is satisfied, we have that $k_{i}^{m} \leq \mu_{-}^{m}+\omega^{m}=$ $2 \mu_{-}^{m}-\mu_{-}^{m}+\omega^{m} \leq 2 \omega^{m}=2 \theta^{\frac{m}{1-m}}$, while in the case (N) we have $k_{i}^{m} \leq \mu_{-}^{m}+\omega^{m}=\mu_{+}^{m} \leq$ $2^{m} \theta^{\frac{m}{1-m}}$. Taking also into account that $\frac{1}{2} \varrho \leq \varrho_{i} \leq \varrho$ for all $i \in \mathbb{N}_{0}$, we find that

$$
Y_{i+1} \leq c b^{i} Y_{i}^{1+\frac{2}{n}}
$$

Note that the constant $c$ depends only on $n, m$. Lemma 3.2 now yields $Y_{i} \rightarrow 0$ as $i \rightarrow \infty$, provided that

$$
Y_{0} \leq c^{-\frac{n}{2}} b^{-\frac{n^{2}}{4}}
$$

This clearly holds if we take $\nu_{o}:=c^{-\frac{n}{2}} b^{-\frac{n^{2}}{4}}$. Note that $\nu_{o}$ depends only on $n$ and $m$. Since $k_{i}^{m} \rightarrow \mu_{-}^{m}+\frac{1}{4} \omega^{m}$ and $\varrho_{i} \rightarrow \frac{1}{2} \varrho$ as $i \rightarrow \infty$, we have thus shown that

$$
\left|Q_{\varrho / 2, \theta(\varrho / 2)^{2}}\left(z_{o}\right) \cap\left\{u^{m}<\mu_{-}^{m}+\frac{1}{4} \omega^{m}\right\}\right|=0,
$$

as desired. 


\subsubsection{The second alternative}

It remains to treat the second alternative considered in Lemma 6.2, when (6.2) is violated. We begin with a lemma that is analogous to Lemma 6.1. However, the methods from the proof of Lemma 6.1 work only for a small parameter $\nu_{1}$ in $(6.7)$ below, while the negation of (6.2) at first yields (6.7) only for $1-\nu_{o}$ instead of $\nu_{1}$, where $\nu_{o}$ is the small constant determined in Lemma 6.1. This is the reason why compared to Lemma 6.1 , in Lemma 6.3 we have to replace the cylinder $Q_{\varrho, \theta \varrho^{2}}\left(z_{o}\right)$ by the smaller cylinder $Q_{\varrho, \frac{1}{2} \nu \theta \varrho^{2}}\left(z_{o}\right)$ with $\nu \in(0,1)$, and why we have to introduce a small parameter $\xi \in\left(0, \frac{1}{2}\right]$ instead of $\frac{1}{2}$. Later on we will show that the opposite of (6.2) implies assumption (6.7) on a smaller cylinder, for some suitable choice of $\xi$. We stress that the constant $\nu_{1}$ in the following lemma does not depend on $\xi$, so that we still have the freedom to choose the latter parameter.

Lemma 6.3. Suppose that the assumptions from the beginning of Section 6.1 and $(6.3)$ are in force. Moreover, let $\nu \in(0,1)$ and $\xi \in\left(0, \frac{1}{2}\right]$. Then, there exists $\nu_{1}=\nu_{1}(n, m, \nu) \in(0,1)$ such that if

$$
\left|Q_{\varrho, \frac{1}{2} \nu \theta \varrho^{2}}\left(z_{o}\right) \cap\left\{u^{m}>\mu_{+}^{m}-\xi \omega^{m}\right\}\right|<\nu_{1}\left|Q_{\varrho, \frac{1}{2} \nu \theta \varrho^{2}}\right|,
$$

then

$$
u^{m} \leq \mu_{+}^{m}-\frac{1}{2} \xi \omega^{m} \quad \text { a.e. in } Q_{\varrho / 2, \frac{1}{2} \nu \theta(\varrho / 2)^{2}}\left(z_{o}\right) .
$$

Proof. For $i \in \mathbb{N}_{0}$ we define

$$
\left(\xi_{i}\right)^{m}:=\frac{1}{2} \xi+\frac{1}{2^{i+1}} \xi, \quad k_{i}^{m}:=\mu_{+}^{m}-\left(\xi_{i} \omega\right)^{m}, \quad \text { and } \quad \varrho_{i}:=\frac{\varrho}{2}+\frac{\varrho}{2^{i+1}}
$$

and

$$
Y_{i}:=\frac{\left|Q_{i} \cap\left\{u>k_{i}\right\}\right|}{\left|Q_{i}\right|}, \quad \text { where } Q_{i}:=Q_{\varrho_{i}, \frac{1}{2} \nu \theta \varrho_{i}^{2}}\left(z_{o}\right)
$$

Note that $\left(\xi_{o}\right)^{m}=\xi, \xi_{i}$ is decreasing and $\left(\xi_{i}\right)^{m} \downarrow \frac{1}{2} \xi$ as $i \rightarrow \infty$. Similarly, we have that $\varrho_{o}=\varrho, \varrho_{i}$ is decreasing and $\varrho_{i} \downarrow \frac{1}{2} \varrho$ as $i \rightarrow \infty$. Note that (6.3) implies $k_{i}^{m} \geq \mu_{+}^{m}-\frac{1}{2} \omega^{m}=$ $\frac{1}{2}\left(\mu_{+}^{m}+\mu_{-}^{m}\right) \geq \sup _{Q_{0}} \psi^{m}$ for any $i \in \mathbb{N}_{0}$. As in the proof of Lemma 6.1 we are going to derive a suitable estimate for $Y_{i+1}$ in terms of $Y_{i}$, and then apply Lemma 3.2. We apply Gagliardo-Nirenberg's inequality from Lemma 3.1 and the energy estimate 
from Lemma 4.1 (i) to get

$$
\begin{aligned}
\int_{Q_{i+1}}\left[u^{m}-k_{i}^{m}\right]_{+}^{\frac{2(n+2)}{n}} & d z \\
\leq & c\left(\sup _{t \in\left(t_{o}-\theta \varrho_{i+1}^{2}, t_{o}\right)} \int_{B_{\varrho_{i+1}}}\left[u^{m}-k_{i}^{m}\right]_{+}^{2} d x\right)^{\frac{2}{n}} \\
& \times \int_{Q_{i+1}}\left(\left|\nabla\left[u^{m}-k_{i}^{m}\right]_{+}\right|^{2}+\left|\frac{\left[u^{m}-k_{i}^{m}\right]_{+}}{\varrho_{i+1}}\right|^{2}\right) d z \\
\leq & c \mu_{+}^{\frac{2(m-1)}{n}}\left(\frac{2^{2 i}}{\varrho^{2}}+\frac{2^{2 i} k_{i}^{1-m}}{\theta \varrho^{2}}\right)^{1+\frac{2}{n}}\left(\int_{Q_{i}}\left[u^{m}-k_{i}^{m}\right]_{+}^{2} d z\right)^{1+\frac{2}{n}} \\
\leq & \frac{c \mu_{+}^{\frac{2(m-1)}{n}}\left(\xi \omega^{m}\right)^{\frac{2(n+2)}{n}} 2^{\frac{2 i(n+2)}{n}}}{\varrho^{\frac{2(n+2)}{n}}}\left(1+\frac{k_{i}^{1-m}}{\theta}\right)^{1+\frac{2}{n}}\left|Q_{i} \cap\left\{u>k_{i}\right\}\right|^{1+\frac{2}{n}},
\end{aligned}
$$

where $c=c(n, m, \nu)$. For the last two inequalities we used the facts that $\mu_{+}^{1-m} \leq u^{1-m}$ and

$$
\left[u^{m}-k_{i}^{m}\right]_{+} \leq \mu_{+}^{m}-k_{i}^{m}=\left(\xi_{i} \omega\right)^{m} \leq \xi \omega^{m},
$$

which holds due to the assumption $\sup _{Q_{0}} u \leq \mu_{+}$. Now, we observe that $k_{i}^{m} \geq \mu_{+}^{m}-$ $\frac{1}{2} \omega^{m} \geq \frac{1}{2} \mu_{+}^{m} \geq \frac{1}{2} \omega^{m}$. Therefore, if (D) is satisfied, we have that $k_{i}^{1-m} \leq 2^{\frac{m-1}{m}} \theta$, while in the case (N) we have $k_{i}^{1-m} \leq 2^{\frac{m-1}{m}} \mu_{+}^{1-m} \leq 2^{\frac{m^{2}-1}{m}} \theta$. Therefore, we arrive at

$$
\int_{Q_{i+1}}\left[u^{m}-k_{i}^{m}\right]_{+}^{\frac{2(n+2)}{n}} d z \leq \frac{c \mu_{+}^{\frac{2(m-1)}{n}}\left(\xi \omega^{m}\right)^{\frac{2(n+2)}{n}} 2^{\frac{2 i(n+2)}{n}}}{\varrho^{\frac{2(n+2)}{n}}}\left|Q_{i} \cap\left\{u>k_{i}\right\}\right|^{1+\frac{2}{n}}
$$

Next, we use the fact that

$$
\left[u^{m}-k_{i}^{m}\right]_{+} \geq k_{i+1}^{m}-k_{i}^{m}=\frac{\xi \omega^{m}}{2^{i+2}} \quad \text { in the set }\left\{u>k_{i+1}\right\}
$$

to get

$$
\begin{aligned}
\int_{Q_{i+1}}\left[u^{m}-k_{i}^{m}\right]_{+}^{\frac{2(n+2)}{n}} d z & \geq \int_{Q_{i+1} \cap\left\{u>k_{i+1}\right\}}\left[u^{m}-k_{i}^{m}\right]_{+}^{\frac{2(n+2)}{n}} d z \\
& \geq \frac{\left(\xi \omega^{m}\right)^{\frac{2(n+2)}{n}}}{4^{\frac{2(n+2)}{n}} 2^{\frac{2 i(n+2)}{n}}}\left|Q_{i+1} \cap\left\{u>k_{i+1}\right\}\right| .
\end{aligned}
$$

Combining the last estimate with (6.8), we get

$$
\left|Q_{i+1} \cap\left\{u>k_{i+1}\right\}\right| \leq \frac{c 4^{\frac{2 i(n+2)}{n}} \mu_{+}^{\frac{2(m-1)}{n}}}{\varrho^{\frac{2(n+2)}{n}}}\left|Q_{i} \cap\left\{u>k_{i}\right\}\right|^{1+\frac{2}{n}},
$$


where, again, $c=c(n, m, \nu)$. Dividing both sides by $\left|Q_{i+1}\right|$ and recalling the definition of $Y_{i}$, we get

$$
Y_{i+1} \leq \frac{c b^{i} \mu_{+}^{\frac{2(m-1)}{n}}\left|Q_{i}\right|^{1+\frac{2}{n}}}{\varrho^{2\left(1+\frac{2}{n}\right)}\left|Q_{i+1}\right|} Y_{i}^{1+\frac{2}{n}}
$$

where we abbreviated $b:=4^{\frac{2(n+2)}{n}}$. If $(\mathrm{D})$ is satisfied, we have that $\mu_{+}^{m}=2 \mu_{+}^{m}-$ $\mu_{+}^{m} \leq 2 \mu_{+}^{m}-2 \mu_{-}^{m}=2 \omega^{m}=2 \theta^{\frac{m}{1-m}}$, while in the case that $(\mathrm{N})$ is satisfied we have $\mu_{+}^{m} \leq$ $2^{m} \theta^{\frac{m}{1-m}}$. Taking also into account that $\frac{1}{2} \varrho \leq \varrho_{i} \leq \varrho$ for all $i \in \mathbb{N}$, we obtain

$$
Y_{i+1} \leq c b^{i} Y_{i}^{1+\frac{2}{n}}
$$

for a constant $c$ depending only on $n, m$, and $\nu$. Lemma 3.2 now yields $Y_{i} \rightarrow 0$ as $i \rightarrow \infty$, provided that

$$
Y_{0} \leq c^{-\frac{n}{2}} b^{-\frac{n^{2}}{4}}
$$

This clearly holds if we take $\nu_{1}:=c^{-\frac{n}{2}} b^{-\frac{n^{2}}{4}}$. Note that $\nu_{1}$ depends only on $n, m, \nu$. Since $k_{i}^{m} \rightarrow \mu_{+}^{m}-\frac{1}{2} \xi \omega^{m}$ and $\varrho_{i} \rightarrow \frac{1}{2} \varrho$ as $i \rightarrow \infty$, we have thus shown that

$$
\left|Q_{\varrho / 2, \frac{1}{2} \nu \theta(\varrho / 2)^{2}}\left(z_{o}\right) \cap\left\{u^{m}>\mu_{+}^{m}-\frac{1}{2} \xi \omega^{m}\right\}\right|=0,
$$

as desired.

In the following proof of Lemma 6.2 our aim will be to apply Lemma 6.3. Thereby, the main difficulty will be to ensure that hypothesis (6.4) of Lemma 6.2 implies assumption (6.7) of Lemma 6.3.

Proof of Lemma 6.2. In the following, we let $\nu \in(0,1)$. We now proceed in several steps.

Step 1. Selecting a "good" time slice. We first observe that $\mu_{-}^{m}+\frac{1}{2} \omega^{m}=\mu_{+}^{m}-$ $\frac{1}{2} \omega^{m}$. Therefore, (6.4) can be rewritten as

$$
\left|Q_{\varrho, \theta \varrho^{2}}\left(z_{o}\right) \cap\left\{u^{m}>\mu_{+}^{m}-\frac{1}{2} \omega^{m}\right\}\right| \leq(1-\nu)\left|Q_{\varrho, \theta \varrho^{2}}\right| .
$$

On the other hand, we have

$$
\begin{aligned}
\left|Q_{\varrho, \theta \varrho^{2}}\left(z_{o}\right) \cap\left\{u^{m}>\mu_{+}^{m}-\frac{1}{2} \omega^{m}\right\}\right| & =\int_{t_{o}-\theta \varrho^{2}}^{t_{o}}\left|B_{\varrho}\left(x_{o}\right) \cap\left\{u^{m}(\cdot, t)>\mu_{+}^{m}-\frac{1}{2} \omega^{m}\right\}\right| d t \\
& \geq \int_{t_{o}-\theta \varrho^{2}}^{t_{o}-\frac{1}{2} \nu \theta \varrho^{2}}\left|B_{\varrho}\left(x_{o}\right) \cap\left\{u^{m}(\cdot, t)>\mu_{+}^{m}-\frac{1}{2} \omega^{m}\right\}\right| d t .
\end{aligned}
$$


By the mean value theorem there exists $t_{1} \in\left[t_{o}-\theta \varrho^{2}, t_{o}-\frac{1}{2} \nu \theta \varrho^{2}\right]$ such that

$$
\begin{aligned}
\left|B_{\varrho}\left(x_{o}\right) \cap\left\{u^{m}\left(\cdot, t_{1}\right)>\mu_{+}^{m}-\frac{1}{2} \omega^{m}\right\}\right| & \leq \frac{1}{\left(1-\frac{1}{2} \nu\right) \theta \varrho^{2}}\left|Q_{\varrho, \theta \varrho^{2}}\left(z_{o}\right) \cap\left\{u^{m}>\mu_{+}^{m}-\frac{1}{2} \omega^{m}\right\}\right| \\
& \leq \frac{1-\nu}{\left(1-\frac{1}{2} \nu\right) \theta \varrho^{2}}\left|Q_{\varrho, \theta \varrho^{2}}\right|=\frac{1-\nu}{1-\frac{1}{2} \nu}\left|B_{\varrho}\right| .
\end{aligned}
$$

Step 2. Expansion in time. Our aim here is to prove that an estimate similar to $(6.9)$ holds for any $t \in\left[t_{1}, t_{o}\right]$. Therefore, we define

$$
k^{m}:=\mu_{+}^{m}-\delta \omega^{m}
$$

with $\delta \in\left(0, \frac{1}{2}\right]$ to be chosen later in dependence on $m$ and $\nu$. Moreover, we let $s_{o} \in \mathbb{N}$ with $s_{o}>1-\frac{\log \delta}{\log 2}>1$ to be fixed later on, so that $2^{1-s}<\delta \leq \frac{1}{2}$ for any $s \geq s_{o}$. Now, let us suppose that

$$
\sup _{B_{\varrho}\left(x_{o}\right) \times\left(t_{1}, t_{o}\right)} u^{m} \geq \mu_{+}^{m}-\frac{\delta}{2} \omega^{m}
$$

The case where (6.10) is not satisfied is easier and will be considered at the end of the proof. We let

$$
H^{m}:=\sup _{B_{\varrho}\left(x_{o}\right) \times\left(t_{1}, t_{o}\right)}\left[u^{m}-k^{m}\right]_{+}
$$

and observe from the definition of $k$ and (6.10) that $\frac{\delta}{2} \omega^{m} \leq H^{m} \leq \delta \omega^{m}$. Since $2^{1-s}<$ $\delta \leq \frac{1}{2}$ for any $s \geq s_{o}$, this implies

$$
\frac{1}{2^{s}} \omega^{m}<H^{m} \leq \frac{1}{2} \omega^{m}, \quad \text { for any } s \geq s_{o}
$$

We now define

$$
\phi(v):=\left[\log \left(\frac{H^{m}}{H^{m}+\frac{1}{2^{s}} \omega^{m}-v}\right)\right]_{+} \quad \text { for } v<H^{m}+\frac{1}{2^{s}} \omega^{m} .
$$

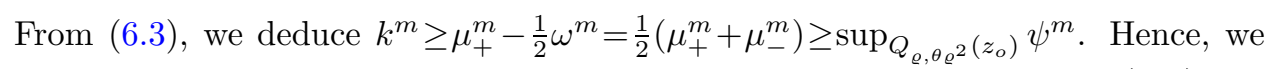
infer from the logarithmic energy estimate in Lemma 4.2 that for any $\sigma \in(0,1)$ and any $t \in\left(t_{1}, t_{o}\right)$ there holds

$$
\begin{aligned}
\mathrm{I}(\mathrm{t}):= & \int_{B_{\sigma \varrho}\left(x_{o}\right) \times\{t\}} u^{1-m} \phi^{2}\left(\left[u^{m}-k^{m}\right]_{+}\right) d x \\
\leq & k^{1-m} \int_{B_{\varrho\left(x_{o}\right)} \times\left\{t_{1}\right\}} \phi^{2}\left(\left[u^{m}-k^{m}\right]_{+}\right) d x \\
& +\frac{8 m}{(1-\sigma)^{2} \varrho^{2}} \int_{B_{\varrho}\left(x_{o}\right) \times\left(t_{1}, t_{o}\right)} \phi\left(\left[u^{m}-k^{m}\right]_{+}\right) d z .
\end{aligned}
$$


Since

$$
\phi\left(\left[u^{m}-k^{m}\right]_{+}\right) \leq \log \left(\frac{2^{s} H^{m}}{\omega^{m}}\right) \leq \log 2^{s-1},
$$

we can use (6.9) (recall that $\left.k^{m} \geq \mu_{+}^{m}-\frac{1}{2} \omega^{m}\right)$ to further estimate

$$
\begin{aligned}
\mathrm{I}(\mathrm{t}) & \leq k^{1-m}\left(\log 2^{s-1}\right)^{2}\left|B_{\varrho}\left(x_{o}\right) \cap\left\{u\left(\cdot, t_{1}\right)>k\right\}\right|+\frac{8 m \log 2^{s-1}}{(1-\sigma)^{2} \varrho^{2}}\left|B_{\varrho} \times\left(t_{1}, t_{o}\right)\right| \\
& \leq\left[k^{1-m}\left(\log 2^{s-1}\right)^{2} \frac{1-\nu}{1-\frac{1}{2} \nu}+\frac{8 m \theta \log 2^{s-1}}{(1-\sigma)^{2}}\right]\left|B_{\varrho}\right|,
\end{aligned}
$$

where in the last line we have also used that $t_{o}-t_{1} \leq \theta \varrho^{2}$. On the other hand, on the set $B_{\sigma \varrho}\left(x_{o}\right) \cap\left\{u^{m}(\cdot, t) \geq \mu_{+}^{m}-\frac{1}{2^{s}} \omega^{m}\right\}$, with $t \in\left(t_{1}, t_{o}\right)$ we have

$$
\left[u^{m}-k^{m}\right]_{+} \geq \mu_{+}^{m}-\frac{1}{2^{s}} \omega^{m}-\left(\mu_{+}^{m}-\delta \omega^{m}\right)=\left(\delta-\frac{1}{2^{s}}\right) \omega^{m} .
$$

Since $\phi\left(\left[u^{m}-k^{m}\right]_{+}\right)$is a decreasing function of $H$ and $H \leq \delta \omega^{m}$, this implies

$$
\begin{aligned}
\phi\left(\left[u^{m}-k^{m}\right]_{+}\right) & \geq\left[\log \left(\frac{\delta \omega^{m}}{\delta \omega^{m}+\frac{1}{2^{s}} \omega^{m}-\left[u^{m}-k^{m}\right]_{+}}\right)\right]_{+} \\
& \geq\left[\log \left(\frac{\delta \omega^{m}}{\delta \omega^{m}+\frac{1}{2^{s}} \omega^{m}-\left(\delta-\frac{1}{2^{s}}\right) \omega^{m}}\right)\right]_{+}=\log \left(2^{s-1} \delta\right) .
\end{aligned}
$$

Therefore, we get the following lower bound for $\mathrm{I}(\mathrm{t})$ :

$$
\mathrm{I}(\mathrm{t}) \geq \mu_{+}^{1-m}\left(\log \left(2^{s-1} \delta\right)\right)^{2}\left|B_{\sigma \varrho}\left(x_{o}\right) \cap\left\{u^{m}(\cdot, t) \geq \mu_{+}^{m}-\frac{1}{2^{s}} \omega^{m}\right\}\right|
$$

for all $t \in\left(t_{1}, t_{o}\right)$. Joining $(6.11)$ and (6.12) yields

$$
\begin{aligned}
\mid B_{\sigma \varrho}\left(x_{o}\right) \cap\left\{u^{m}(\cdot, t) \geq\right. & \left.\mu_{+}^{m}-\frac{1}{2^{s}} \omega^{m}\right\} \mid \\
& \leq \frac{\mu_{+}^{m-1}}{\left(\log \left(2^{s-1} \delta\right)\right)^{2}}\left[k^{1-m}\left(\log 2^{s-1}\right)^{2} \frac{1-\nu}{1-\frac{1}{2} \nu}+\frac{8 m \theta \log 2^{s-1}}{(1-\sigma)^{2}}\right]\left|B_{\varrho}\right|,
\end{aligned}
$$

for all $t \in\left(t_{1}, t_{o}\right)$. At this point, we use that $k^{m}=(1-\delta) \mu_{+}^{m}+\delta \mu_{-}^{m} \geq(1-\delta) \mu_{+}^{m}$. Moreover, if (D) is satisfied, then $\omega^{m}=\mu_{+}^{m}-\mu_{-}^{m} \geq \frac{1}{2} \mu_{+}^{m}$ and hence $\theta \leq 2 \mu_{+}^{1-m}$, while in the case $(\mathrm{N})$ we have $\theta \leq 2^{m-1} \mu_{+}^{1-m}$. Taking also into account that $\left|B_{\varrho} \backslash B_{\sigma \varrho}\right|=$ $\left(1-\sigma^{n}\right)\left|B_{\varrho}\right| \leq n(1-\sigma)\left|B_{\varrho}\right|$, we can further estimate

$$
\begin{aligned}
\mid B_{\varrho}\left(x_{o}\right) \cap & \left\{u^{m}(\cdot, t) \geq \mu_{+}^{m}-\frac{1}{2^{s}} \omega^{m}\right\} \mid \\
& \leq\left[(1-\delta)^{\frac{1-m}{m}}\left(\frac{\log 2^{s-1}}{\log \left(2^{s-1} \delta\right)}\right)^{2} \frac{1-\nu}{1-\frac{1}{2} \nu}+\frac{2^{m+3} m \log 2^{s-1}}{(1-\sigma)^{2}\left(\log \left(2^{s-1} \delta\right)\right)^{2}}+n(1-\sigma)\right]\left|B_{\varrho}\right| .
\end{aligned}
$$


We now choose

$$
\sigma:=1-\frac{\nu^{2}}{8 n} \in(0,1)
$$

and

$$
\delta:=\min \left\{\frac{1}{2}, 1-\left(\frac{1-\nu^{2}}{1-\frac{1}{2} \nu^{2}}\right)^{\frac{m}{m-1}}\right\} \leq \frac{1}{2}
$$

and note that

$$
(1-\delta)^{\frac{1-m}{m}} \leq \frac{1-\frac{1}{2} \nu^{2}}{1-\nu^{2}}
$$

Then, the last inequality yields that

$$
\begin{aligned}
\mid B_{\varrho}\left(x_{o}\right) \cap\left\{u^{m}(\cdot, t)\right. & \left.\geq \mu_{+}^{m}-\frac{1}{2^{s}} \omega^{m}\right\} \mid \\
& \leq\left[\left(\frac{\log 2^{s-1}}{\log \left(2^{s-1} \delta\right)}\right)^{2} \frac{1-\frac{1}{2} \nu^{2}}{\left(1-\frac{1}{2} \nu\right)(1+\nu)}+\frac{2^{m+9} n^{2} m \log 2^{s-1}}{\nu^{4}\left(\log \left(2^{s-1} \delta\right)\right)^{2}}+\frac{\nu^{2}}{8}\right]\left|B_{\varrho}\right|,
\end{aligned}
$$

for any $t \in\left(t_{1}, t_{o}\right)$. Next, we choose $s_{o}$ in dependence on $n, m$ and $\nu$ large enough to ensure that

$$
\left(\frac{\log 2^{s_{o}-1}}{\log \left(2^{s_{o}-1} \delta\right)}\right)^{2} \leq(1+\nu)\left(1-\frac{1}{2} \nu\right)
$$

and

$$
\frac{2^{m+9} n^{2} m \log 2^{s_{o}-1}}{\left(\log \left(2^{s_{o}-1} \delta\right)\right)^{2}} \leq \frac{\nu^{6}}{8}
$$

holds true. Then, we have for any $s \geq s_{o}$ and any $t \in\left(t_{1}, t_{o}\right)$ that

$$
\left|B_{\varrho}\left(x_{o}\right) \cap\left\{u^{m}(\cdot, t) \geq \mu_{+}^{m}-\frac{1}{2^{s}} \omega^{m}\right\}\right| \leq\left(\left(1-\frac{1}{2} \nu^{2}\right)+\frac{1}{4} \nu^{2}\right)\left|B_{\varrho}\right|=\left(1-\frac{1}{4} \nu^{2}\right)\left|B_{\varrho}\right|,
$$

provided that (6.10) is satisfied. On the other hand, if (6.10) is not satisfied, then we have that

$$
\left|B_{\varrho}\left(x_{o}\right) \cap\left\{u^{m}(\cdot, t) \geq \mu_{+}^{m}-\frac{\delta}{2} \omega^{m}\right\}\right|=0
$$

holds true for any $t \in\left[t_{1}, t_{o}\right]$. Since $\frac{\delta}{2}>\frac{1}{2^{s}}$ for any $s \geq s_{o}$, this implies the second last inequality. Therefore, in any case we have proved that there exists $s_{o}=s_{o}(n, m, \nu) \in$ $\mathbb{N}_{\geq 2}$ such that

$$
\left|B_{\varrho}\left(x_{o}\right) \cap\left\{u^{m}(\cdot, t) \geq \mu_{+}^{m}-\frac{1}{2^{s}} \omega^{m}\right\}\right| \leq\left(1-\frac{1}{4} \nu^{2}\right)\left|B_{\varrho}\right|,
$$

or equivalently

$$
\left|B_{\varrho}\left(x_{o}\right) \cap\left\{u^{m}(\cdot, t)<\mu_{+}^{m}-\frac{1}{2^{s}} \omega^{m}\right\}\right| \geq \frac{1}{4} \nu^{2}\left|B_{\varrho}\right|,
$$

holds true for any $t \in\left[t_{1}, t_{o}\right]$ and any $s \geq s_{o}$. 
Step 3. Improving the measure estimate on a smaller cylinder. Let $\nu_{1}=$ $\nu_{1}(n, m, \nu)$ be the corresponding parameter from Lemma 6.3. Here, we will prove that there exists $s_{1}=s_{1}(n, m, \nu) \in \mathbb{N}_{\geq 2}$ such that

$$
\left|Q_{\varrho, \frac{1}{2} \nu \theta \varrho^{2}}\left(z_{o}\right) \cap\left\{u^{m}>\mu_{+}^{m}-\frac{1}{2^{s_{o}+s_{1}}} \omega^{m}\right\}\right|<\nu_{1}\left|Q_{\varrho, \frac{1}{2} \nu \theta \varrho^{2}}\right| .
$$

We abbreviate $Q_{2}:=B_{\varrho}\left(x_{o}\right) \times\left(t_{o}-\frac{1}{2} \nu \theta \varrho^{2}, t_{o}\right]$ and $Q_{1}:=B_{2 \varrho}\left(x_{o}\right) \times\left(t_{o}-\nu \theta \varrho^{2}, t_{o}\right]$, so that $Q_{2} \subset Q_{1} \subset Q_{2 \varrho, \theta(2 \varrho)^{2}}\left(z_{o}\right)$. For $j \in \mathbb{N}$ we define

$$
\left(k_{j}\right)^{m}:=\mu_{+}^{m}-\frac{1}{2^{j}} \omega^{m}
$$

and the associated superlevel sets

$$
A_{j}:=Q_{2} \cap\left\{u>k_{j}\right\} .
$$

An application of Lemma 3.3 to $u^{m}(\cdot, t)$ on $B_{\varrho}\left(x_{o}\right)$ with $t \in\left(t_{o}-\frac{1}{2} \nu \theta \varrho^{2}, t_{o}\right)$ and $\ell, k$ replaced by $k_{j+1}^{m}, k_{j}^{m}$ yields that

$$
\begin{aligned}
\left(k_{j+1}^{m}-k_{j}^{m}\right) \mid B_{\varrho}\left(x_{o}\right) \cap\{ & \left.u^{m}(\cdot, t)>k_{j+1}^{m}\right\} \mid \\
& \leq \frac{c(n) \varrho^{n+1}}{\left|B_{\varrho}\left(x_{o}\right) \cap\left\{u^{m}(\cdot, t)<k_{j}^{m}\right\}\right|} \int_{B_{\varrho}\left(x_{o}\right) \cap\left\{k_{j}<u(\cdot, t)<k_{j+1}\right\}}\left|\nabla u^{m}\right| d x \\
& \leq \frac{c(n) \varrho}{\nu^{2}} \int_{B_{\varrho}\left(x_{o}\right) \cap\left\{k_{j}<u(\cdot, t)<k_{j+1}\right\}}\left|\nabla u^{m}\right| d x,
\end{aligned}
$$

for any $j \in \mathbb{N}$ with $j \geq s_{o}$. We have also used (6.14) and the fact that $\left(t_{o}-\frac{1}{2} \nu \theta \varrho^{2}, t_{o}\right] \subset$ $\left[t_{1}, t_{o}\right]$ in the last line. We integrate the last inequality with respect to $t$ over $\left(t_{o}-\frac{1}{2} \nu \theta \varrho^{2}, t_{o}\right)$ to get

$$
\begin{aligned}
\left(k_{j+1}^{m}-k_{j}^{m}\right)\left|A_{j+1}\right| & \leq \frac{c(n) \varrho}{\nu^{2}} \int_{A_{j} \backslash A_{j+1}}\left|\nabla u^{m}\right| d z \\
& \leq \frac{c(n) \varrho}{\nu^{2}}\left|A_{j} \backslash A_{j+1}\right|^{\frac{1}{2}}\left(\int_{A_{j} \backslash A_{j+1}}\left|\nabla u^{m}\right|^{2} d z\right)^{\frac{1}{2}} \\
& \leq \frac{c(n) \varrho}{\nu^{2}}\left|A_{j} \backslash A_{j+1}\right|^{\frac{1}{2}}\left(\int_{Q_{2}}\left|\nabla\left[u^{m}-k_{j}^{m}\right]_{+}\right|^{2} d z\right)^{\frac{1}{2}} .
\end{aligned}
$$

Note that from $(6.3)$ we have $\left(k_{j}\right)^{m} \geq \mu_{+}^{m}-\frac{1}{2} \omega^{m}=\frac{1}{2}\left(\mu_{+}^{m}+\mu_{-}^{m}\right) \geq \sup _{Q_{2 \varrho, \theta(2 \varrho)^{2}}\left(z_{o}\right)} \psi^{m}$ for any $j \in \mathbb{N}$. Therefore, the energy estimate in Lemma 4.1 (i) implies

$$
\int_{Q_{2}}\left|\nabla\left[u^{m}-k_{j}^{m}\right]_{+}\right|^{2} d z \leq c(m)\left(\frac{1}{\varrho^{2}}+\frac{k_{j}^{1-m}}{\nu \theta \varrho^{2}}\right) \int_{Q_{1}}\left[u^{m}-k_{j}^{m}\right]_{+}^{2} d z .
$$


First, we observe that $k_{j}^{m}=\mu_{+}^{m}-2^{-j} \omega^{m} \geq \frac{1}{2} \mu_{+}^{m} \geq \frac{1}{2} \omega^{m}$. Therefore, if (D) is satisfied, we have that $k_{j}^{1-m} \leq 2^{\frac{m-1}{m}} \theta$, while in the case $(\mathrm{N})$ we have $k_{j}^{1-m} \leq 2^{\frac{m-1}{m}} \mu_{+}^{1-m} \leq$ $2^{\frac{m^{2}-1}{m}} \theta$. Using also the fact that $\left[u^{m}-k_{j}^{m}\right]_{+} \leq \mu_{+}^{m}-k_{j}^{m}=2^{-j} \omega^{m}$ on $Q_{2}$, we can further estimate

$$
\int_{Q_{2}}\left|\nabla\left[u^{m}-k_{j}^{m}\right]_{+}\right|^{2} d z \leq \frac{c(m)}{\nu \varrho^{2}}\left(\frac{\omega^{m}}{2^{j}}\right)^{2}\left|Q_{1}\right| .
$$

Inserting this estimate above and taking into account that $k_{j+1}^{m}-k_{j}^{m}=2^{-(j+1)} \omega^{m}$, we obtain

$$
\left|A_{j+1}\right|^{2} \leq \frac{c(n, m)}{\nu^{5}}\left|A_{j} \backslash A_{j+1}\right|\left|Q_{1}\right| .
$$

Now, we let $s_{1} \in \mathbb{N}_{\geq 2}$ and add up the preceding inequality for $j=s_{o}, \ldots, s_{o}+s_{1}-1$ to infer that

$$
\left(s_{1}-1\right)\left|A_{s_{o}+s_{1}}\right|^{2} \leq \frac{c(n, m)}{\nu^{5}}\left|Q_{1}\right|^{2}=\frac{c(n, m)}{\nu^{5}}\left|Q_{2}\right|^{2} .
$$

Choosing $s_{1}=s_{1}\left(n, m, \nu, \nu_{1}\right) \equiv s_{1}(n, m, \nu)$ large enough to ensure that

$$
\frac{c(n, m)}{\nu^{5}\left(s_{1}-1\right)} \leq \nu_{1}^{2}
$$

we conclude the claim (6.15).

Step 4. Concluding the proof of Lemma 6.2. Due to (6.15) we are allowed to apply Lemma 6.3 with $\xi=2^{-\left(s_{o}+s_{1}\right)}$ to conclude that

$$
u^{m} \leq \mu_{+}^{m}-\frac{1}{2} \xi \omega^{m} \quad \text { a.e. in } Q_{\varrho / 2, \frac{1}{2} \nu \theta(\varrho / 2)^{2}}\left(z_{o}\right) .
$$

This proves the assertion of Lemma 6.2 for the choice $a=\frac{1}{2} \xi$. Note that $\xi$ depends on $n, m, \nu$ and therefore the parameter $a$ depends on the same quantities.

Combining the two alternatives (Lemma 6.1 and Lemma 6.2) we get the following proposition.

Proposition 6.4. Suppose that the assumptions from the beginning of Section 6.1 are in force and let $Q_{2 \varrho, \theta(2 \varrho)^{2}}\left(z_{o}\right) \subset \Omega_{T}$ be a parabolic cylinder satisfying

$$
\inf _{Q_{2 \varrho, \theta(2 \varrho)^{2}}\left(z_{o}\right)} u \geq \mu_{-}, \quad \sup _{Q_{2 \varrho, \theta(2 \varrho)^{2}}\left(z_{o}\right)} u \leq \mu_{+}, \quad \text { and } \sup _{Q_{2 \varrho, \theta(2 \varrho)^{2}}\left(z_{o}\right)} \psi^{m} \leq \frac{1}{2}\left(\mu_{+}^{m}+\mu_{-}^{m}\right) .
$$

Then, there exists $\nu_{o}=\nu_{o}(n, m) \in(0,1)$ and $a=a(n, m) \in\left(0, \frac{1}{4}\right]$ such that either

$$
\inf _{Q_{\varrho / 2, \theta(\varrho / 2)^{2}}\left(z_{o}\right)} u^{m} \geq \mu_{-}^{m}+\frac{1}{4} \omega^{m}, \quad \text { or } \sup _{Q_{\varrho / 2, \frac{1}{2} \nu_{o} \theta(\varrho / 2)^{2}}\left(z_{o}\right)} u^{m} \leq \mu_{+}^{m}-a \omega^{m}
$$

holds true. 
Proof. We let $\nu_{o}=\nu_{o}(n, m) \in(0,1)$ be the constant from Lemma 6.1. Then we take $a=a\left(n, m, \nu_{o}\right) \equiv a(n, m) \in\left(0, \frac{1}{4}\right]$ to be the constant from Lemma 6.2 applied with $\nu=\nu_{o}$. With these choices, one of the alternatives (6.2) and (6.4) of Lemmas 6.1 and 6.2 is satisfied for the given cylinder. Therefore, the application of Lemma 6.1, respectively Lemma 6.2 yields the claim.

\subsection{The degenerate and the nondegenerate regime}

In this section we construct smaller cylinders on which the oscillation is reduced. We need to treat the two different regimes introduced at the beginning of Section 6.1 separately.

Throughout this subsection we let $\nu_{o}=\nu_{o}(n, m) \in(0,1)$ and $a=a(n, m) \in\left(0, \frac{1}{4}\right]$ be the constants from Proposition 6.4 and define $\delta:=1-a \in\left[\frac{3}{4}, 1\right)$.

We start by considering the degenerate regime. Here, we prove a reduction of the oscillation of $u^{m}$ on a smaller cylinder. Since we do not know if the smaller cylinder again belongs to the degenerate regime, we cannot iterate the argument.

Proposition 6.5. Let $\mu_{-}, \mu_{+} \geq 0$ be two parameters with

$$
\mu_{-} \leq \frac{1}{2} \mu_{+}
$$

and define

$$
\theta:=\omega^{1-m} \quad \text { with } \omega^{m}:=\mu_{+}^{m}-\mu_{-}^{m} .
$$

Suppose that $Q:=Q_{\varrho, \theta \varrho^{2}}\left(z_{o}\right) \subset \Omega_{T}$ is a parabolic cylinder satisfying

$$
\inf _{Q} u \geq \mu_{-}, \quad \sup _{Q} u \leq \mu_{+}, \quad \text { and } \quad \sup _{Q} \psi^{m} \leq \frac{1}{2}\left(\mu_{+}^{m}+\mu_{-}^{m}\right) .
$$

Then, with

$$
\left(\omega_{1}\right)^{m}:=\max \left\{\delta \omega^{m}, 2 \underset{Q}{\operatorname{osc}} \psi^{m}\right\}
$$

and

$$
Q_{1}:=Q_{\varrho_{1}, \theta_{1} \varrho_{1}^{2}}\left(z_{o}\right), \quad \text { where } \theta_{1}:=\omega_{1}^{1-m}, \varrho_{1}:=\eta \varrho, \eta:=\sqrt{\frac{1}{32} \nu_{o} \delta^{\frac{m-1}{m}}}
$$

there holds

$$
\underset{Q_{1}}{\operatorname{Osc}} u^{m} \leq \omega_{1}^{m}, \quad \text { and } \quad Q_{1} \subset Q .
$$


Proof. First, we observe that $\eta<\frac{1}{4} \delta^{\frac{m-1}{2 m}}<\frac{1}{4}$ and $\omega_{1} \geq \delta^{\frac{1}{m}} \omega$, which implies that

$$
Q_{1} \subset Q_{\varrho / 4, \frac{1}{2} \nu_{o} \theta(\varrho / 4)^{2}}\left(z_{o}\right) \subset Q_{\varrho / 4, \theta(\varrho / 4)^{2}}\left(z_{o}\right) \subset Q .
$$

Moreover, we note that assumption (D) is satisfied. Therefore, we can apply Proposition 6.4 to conclude that either

$$
\inf _{Q_{\varrho / 4, \theta(\varrho / 4)^{2}}\left(z_{o}\right)} u^{m} \geq \mu_{-}^{m}+\frac{1}{4} \omega^{m}, \quad \text { or } \quad \sup _{Q_{\varrho / 4, \frac{1}{2} \nu_{o} \theta(\varrho / 4)^{2}}\left(z_{o}\right)} u^{m} \leq \mu_{+}^{m}-a \omega^{m}
$$

holds true. By (6.18), this implies that either

$$
\inf _{Q_{1}} u^{m} \geq \mu_{-}^{m}+\frac{1}{4} \omega^{m}, \quad \text { or } \quad \sup _{Q_{1}} u^{m} \leq \mu_{+}^{m}-a \omega^{m}
$$

is satisfied. If the first alternative occurs, we conclude

$$
\underset{Q_{1}}{\operatorname{Osc}} u^{m}=\sup _{Q_{1}} u^{m}-\inf _{Q_{1}} u^{m} \leq \mu_{+}^{m}-\left(\mu_{-}^{m}+\frac{1}{4} \omega^{m}\right)=\left(1-\frac{1}{4}\right) \omega^{m} \leq \delta \omega^{m} \leq \omega_{1}^{m},
$$

while in the case that the second alternative occurs, we have that

$$
\underset{Q_{1}}{\operatorname{OSc}} u^{m}=\sup _{Q_{1}} u^{m}-\inf _{Q_{1}} u^{m} \leq \mu_{+}^{m}-a \omega^{m}-\mu_{-}^{m}=(1-a) \omega^{m}=\delta \omega^{m} \leq \omega_{1}^{m} .
$$

This proves the assertion of the proposition.

Next, we consider the nondegenerate regime. As in the degenerate regime, we can prove a reduction of the oscillation on a smaller cylinder. However, in contrast to the degenerate regime, we can even prove that the smaller cylinder again belongs to the nondegenerate regime. Therefore, we can use an induction argument to prove a reduction of the oscillation on a sequence of concentric nested cylinders.

Proposition 6.6. Let $\nu_{o}=\nu_{o}(n, m) \in(0,1)$ and $\delta=\delta(n, m) \in(0,1)$ be the constants from the beginning of Section 6.2 and let $0<\mu_{-} \leq \mu_{+}$be two parameters satisfying

$$
\mu_{-}>\frac{1}{2} \mu_{+}
$$

and define $\theta:=\mu_{+}^{1-m}$. Suppose that $Q_{o}:=Q_{\varrho_{o}, \theta \varrho_{o}^{2}}\left(z_{o}\right) \subset \Omega_{T}$ is a parabolic cylinder satisfying

$$
\inf _{Q_{o}} u \geq \mu_{-} \quad \text { and } \quad \sup _{Q_{o}} u \leq \mu_{+},
$$

as well as

$$
\sup _{Q_{o}} \psi^{m} \leq \frac{1}{2}\left(\mu_{+}^{m}+\mu_{-}^{m}\right) \quad \text { and } \quad \underset{Q_{o}}{\operatorname{osc}} \psi^{m} \leq \frac{1}{2}\left(\mu_{+}^{m}-\mu_{-}^{m}\right) .
$$


With the sequence of cylinders

$$
Q_{i}:=Q_{\varrho_{i}, \theta \varrho_{i}^{2}}\left(z_{o}\right), \quad \text { where } \varrho_{i}:=\eta^{i} \varrho_{o}, \eta:=\sqrt{\frac{1}{32} \nu_{o}},
$$

we define

$$
\left(\omega_{o}\right)^{m}:=\mu_{+}^{m}-\mu_{-}^{m}, \quad \text { and } \quad\left(\omega_{i}\right)^{m}:=\max \left\{\delta \omega_{i-1}^{m}, 2 \underset{Q_{i-1}}{\operatorname{osc}} \psi^{m}\right\} \text { for } i \in \mathbb{N} .
$$

Then, for any $i \in \mathbb{N}_{0}$ there holds

$$
\underset{Q_{i}}{\operatorname{OSc}} u^{m} \leq \omega_{i}^{m}
$$

Proof. First, we observe that

$$
Q_{i+1} \subset Q_{\varrho_{i} / 4, \frac{1}{2} \nu_{o} \theta\left(\varrho_{i} / 4\right)^{2}}\left(z_{o}\right) \subset Q_{\varrho_{i} / 4, \theta\left(\varrho_{i} / 4\right)^{2}}\left(z_{o}\right) \subset Q_{i}, \quad \text { for any } i \in \mathbb{N}_{0} .
$$

Next, we define $\mu_{-, o}:=\mu_{-}$and $\mu_{+, o}:=\mu_{+}$, as well as

$$
\mu_{-, i}:=\inf _{Q_{i}} u, \quad \mu_{+, i}^{m}:=\mu_{-, i}^{m}+\omega_{i}^{m}, \quad \text { for } i \in \mathbb{N} .
$$

From (6.19) and the definition of $\omega_{1}$, we deduce $\omega_{1} \leq \omega_{o}$. Then, we infer inductively that

$$
\omega_{i+1} \leq \omega_{i} \quad \text { for all } i \in \mathbb{N}_{0}
$$

Next, we note that

$$
\left(2 \mu_{+, i}\right)^{1-m} \leq \theta \leq\left(\frac{1}{2} \mu_{+, i}\right)^{1-m}
$$

holds for any $i \in \mathbb{N}_{0}$. In fact, the first inequality is a consequence of $\theta=\mu_{+, o}^{1-m} \geq$ $\left(2 \mu_{-, o}\right)^{1-m} \geq\left(2 \mu_{-, i}\right)^{1-m} \geq\left(2 \mu_{+, i}\right)^{1-m}$, while the second follows from $\mu_{+, i}^{m}=\mu_{-, i}^{m}+$ $\omega_{i}^{m} \leq \sup _{Q_{o}} u^{m}+\omega_{o}^{m} \leq 2 \mu_{+, o}^{m}=2 \theta^{\frac{m}{1-m}}$. Moreover, we have for any $i \in \mathbb{N}$

$$
\sup _{Q_{i}} \psi^{m}=\inf _{Q_{i}} \psi^{m}+\underset{Q_{i}}{\operatorname{osc}} \psi^{m} \leq \inf _{Q_{i}} u^{m}+\underset{Q_{i-1}}{\operatorname{osc}} \psi^{m} \leq \mu_{-, i}^{m}+\frac{1}{2} \omega_{i}^{m}=\frac{1}{2}\left(\mu_{+, i}^{m}+\mu_{-, i}^{m}\right),
$$

while for $i=0$, the same holds by assumption $(6.19)_{1}$.

In the following, we will prove

$$
\left\{\begin{array}{l}
\mu_{-, i}>\frac{1}{2} \mu_{+, i} \\
\operatorname{osc}_{Q_{i}} u^{m} \leq \omega_{i}^{m},
\end{array}\right.
$$


for any $i \in \mathbb{N}_{0}$ by induction. For $i=0$ the assertion (6.24) is a direct consequence of the assumptions on $\mu_{-}$and $\mu_{+}$. We now assume that (6.24) is satisfied for some $i \in \mathbb{N}_{0}$. Keeping in mind (6.22) and $\mu_{-, i} \leq \mu_{-, i+1}$, we deduce

$$
\begin{aligned}
\mu_{+, i+1}^{m} & =\mu_{-, i+1}^{m}+\omega_{i+1}^{m}=2^{m} \mu_{-, i+1}^{m}+\left(\omega_{i+1}^{m}-\left(2^{m}-1\right) \mu_{-, i+1}^{m}\right) \\
& \leq 2^{m} \mu_{-, i+1}^{m}+\left(\omega_{i}^{m}-\left(2^{m}-1\right) \mu_{-, i}^{m}\right)=2^{m} \mu_{-, i+1}^{m}+\left(\mu_{+, i}^{m}-2^{m} \mu_{-, i}^{m}\right) \\
& <2^{m} \mu_{-, i+1}^{m},
\end{aligned}
$$

which proves the first assertion in (6.24) for $i+1$. Moreover, we have that $\mu_{+, i}^{m}=$ $\mu_{-, i}^{m}+\omega_{i}^{m} \geq \sup _{Q_{i}} u^{m}$ and hence $\mu_{+, i} \geq \sup _{Q_{i}} u$ and assumption (N) holds for $Q_{i}$ by $(6.24)_{1}$ and (6.23). Therefore, we can apply Proposition 6.4 to conclude that either

$$
\inf _{Q_{\varrho_{i} / 4, \theta\left(\varrho_{i} / 4\right)^{2}}\left(z_{o}\right)} u^{m} \geq \mu_{-, i}^{m}+\frac{1}{4} \omega_{i}^{m}, \quad \text { or } \quad \sup _{Q_{\varrho_{i} / 4, \frac{1}{2} \nu_{o} \theta\left(\varrho_{i} / 4\right)^{2}}\left(z_{o}\right)} u^{m} \leq \mu_{+, i}^{m}-a \omega_{i}^{m}
$$

holds true. By the inclusions (6.21), this implies that either

$$
\inf _{Q_{i+1}} u^{m} \geq \mu_{-, i}^{m}+\frac{1}{4} \omega_{i}^{m}, \quad \text { or } \quad \sup _{Q_{i+1}} u^{m} \leq \mu_{+, i}^{m}-a \omega_{i}^{m}
$$

holds true. If the first alternative occurs, we conclude

$$
\begin{aligned}
\underset{Q_{i+1}}{\operatorname{OSc}} u^{m} & =\sup _{Q_{i+1}} u^{m}-\inf _{Q_{i+1}} u^{m} \leq \mu_{+, i}^{m}-\left(\mu_{-, i}^{m}+\frac{1}{4} \omega_{i}^{m}\right) \\
& =\left(1-\frac{1}{4}\right) \omega_{i}^{m} \leq \delta \omega_{i}^{m} \leq \omega_{i+1}^{m}
\end{aligned}
$$

while in the case that the second alternative occurs, we have that

$$
\begin{aligned}
\underset{Q_{i+1}}{\operatorname{OSc}} u^{m} & =\sup _{Q_{i+1}} u^{m}-\inf _{Q_{i+1}} u^{m} \leq \mu_{+, i}^{m}-a \omega_{i}^{m}-\mu_{-, i}^{m} \\
& =(1-a) \omega_{i}^{m}=\delta \omega_{i}^{m} \leq \omega_{i+1}^{m} .
\end{aligned}
$$

Hence, in both cases we have proved $(6.24)_{2}$ and thereby $(6.20)$ for $i+1$. This finishes the proof of the proposition.

\subsection{The final iteration}

In this section we finally prove the Hölder continuity of the solution to the obstacle problem for the porous medium equation. For this aim, we let

$$
\varepsilon:=\frac{2 \beta(m-1)}{2 m+\beta(m-1)} \in(0,1) \quad \text { and } \quad \gamma_{o}:=\frac{2 \beta m}{2 m+\beta(m-1)} \in(0,1),
$$


where $\beta \in(0,1)$ is the Hölder exponent from assumption (6.1). Then, we fix $z_{o} \in \Omega_{T}$ and consider $R \in(0,1)$ such that $Q_{2 R, 4 R^{2-\varepsilon}}\left(z_{o}\right) \subset \Omega_{T}$. If

$$
\underset{Q_{\varrho, \varrho^{2-\varepsilon}}\left(z_{o}\right)}{\operatorname{OSc}} u^{m} \leq \max \left\{\varrho^{\frac{\varepsilon m}{m-1}}, 2 \underset{Q_{\varrho, \varrho^{2-\varepsilon}}\left(z_{o}\right)}{\operatorname{osc}} \psi^{m}\right\}=: \Psi(\varrho), \quad \text { for any } \varrho \in(0, R],
$$

we use the Hölder continuity assumption (6.1) on $\psi$ and the choice of $\varepsilon$ to see that

$$
\Psi(\varrho) \leq c \max \left\{\varrho^{\frac{\varepsilon m}{m-1}}, \varrho^{\beta-\frac{\varepsilon \beta}{2}}\right\}=c \varrho^{\gamma_{o}}, \quad \text { for any } \varrho \in(0, R]
$$

so that $u^{m}$ is Hölder continuous at $z_{o}$. Therefore, it remains to consider the remaining case. In this case we either have for $\varrho_{o}=R$ that

$$
\Psi\left(\varrho_{o}\right)<\underset{Q_{\varrho_{o}, \varrho_{o}^{2-\varepsilon}}\left(z_{o}\right)}{\operatorname{osc}} u^{m} \leq \frac{\left\|u^{m}\right\|_{L^{\infty}}}{R^{\gamma_{o}}} \varrho_{o}^{\gamma_{o}},
$$

or we can find a $\varrho_{o} \in(0, R)$ with

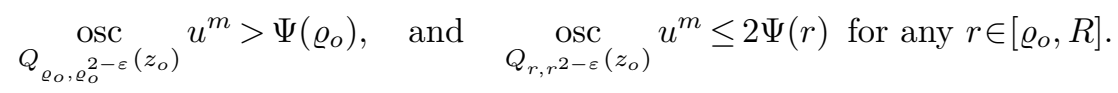

With this choice of $\varrho_{o}$, we define

$$
\mu_{-, o}:=\inf _{Q_{\varrho_{o}, \varrho_{o}^{2-\varepsilon}}\left(z_{o}\right)} u, \quad \mu_{+, o}:=\sup _{Q_{\varrho_{o}, \varrho_{o}^{2-\varepsilon}}\left(z_{o}\right)} u
$$

and

$$
\omega_{o}^{m}:=\mu_{+, o}^{m}-\mu_{-, o}^{m} \quad \text { and } \quad \theta_{o}:=\omega_{o}^{1-m} .
$$

With these choices of parameters, we have

$$
\theta_{o}=\omega_{o}^{1-m}=\left(\begin{array}{c}
\operatorname{osc} \\
Q_{\varrho_{o}, \varrho_{o}^{2-\varepsilon}}\left(z_{o}\right)
\end{array} u^{m}\right)^{\frac{1-m}{m}}<\Psi\left(\varrho_{o}\right)^{\frac{1-m}{m}} \leq \varrho_{o}^{-\varepsilon},
$$

so that $Q_{o}:=Q_{\varrho_{o}, \theta_{o} \varrho_{o}^{2}}\left(z_{o}\right) \subset Q_{\varrho_{o}, \varrho_{o}^{2-\varepsilon}}\left(z_{o}\right)$. Therefore, we find that

$$
\begin{aligned}
\sup _{Q_{o}} \psi^{m} & \leq \sup _{Q_{\varrho_{o}, \varrho_{o}^{2-\varepsilon}}\left(z_{o}\right)} \psi^{m}=\inf _{Q_{\varrho_{o}, \varrho_{o}^{2-\varepsilon}}^{2-\varepsilon}\left(z_{o}\right)} \psi^{m}+\underset{Q_{\varrho_{o}, \varrho_{o}^{2-\varepsilon}}^{2-\varepsilon}\left(z_{o}\right)}{\operatorname{osc}} \psi^{m} \\
& \leq \inf _{Q_{\varrho_{o}, \varrho_{o}^{2-\varepsilon}}\left(z_{o}\right)} u^{m}+\frac{1}{2} \underset{Q_{\varrho_{o}, \varrho_{o}^{2-\varepsilon}}^{2-\varepsilon}\left(z_{o}\right)}{\operatorname{osc}} u^{m}=\mu_{-, o}^{m}+\frac{1}{2}\left(\mu_{+, o}^{m}-\mu_{-, o}^{m}\right) \\
& =\frac{1}{2}\left(\mu_{+, o}^{m}+\mu_{-, o}^{m}\right) .
\end{aligned}
$$

By $\nu_{o}, \delta \in(0,1)$ we denote the corresponding constants from the beginning of Section 6.2 , both depending only on $n$ and $m$. We define sequences of nonnegative 
numbers $\omega_{i}, \mu_{+, i}, \mu_{-, i}$, and cylinders $Q_{i}$ for $i \in \mathbb{N}$ by the following recursive scheme. Assuming that $\omega_{i-1}$ and $Q_{i-1}$ have already been defined, we let

$$
\left(\omega_{i}\right)^{m}:=\max \left\{\delta \omega_{i-1}^{m}, 2 \underset{Q_{i-1}}{\operatorname{osc}} \psi^{m}\right\},
$$

and

$$
\mu_{-, i}:=\inf _{Q_{i}} u, \quad \mu_{+, i}^{m}:=\mu_{-, i}^{m}+\omega_{i}^{m},
$$

as well as

$$
Q_{i}:=Q_{\varrho_{i}, \theta_{i} \varrho_{i}^{2}}\left(z_{o}\right), \quad \text { with } \theta_{i}:=\omega_{i}^{1-m}, \varrho_{i}:=\eta^{i} \varrho_{o}, \eta:=\sqrt{\frac{1}{32} \nu_{o} \delta^{\frac{m-1}{m}}} .
$$

For any $i \in \mathbb{N}$, we deduce

$$
\sup _{Q_{i}} \psi^{m}=\inf _{Q_{i}} \psi^{m}+\operatorname{osc}_{Q_{i}} \psi^{m} \leq \inf _{Q_{i}} u^{m}+\frac{1}{2} \omega_{i}^{m}=\frac{1}{2}\left(\mu_{+, i}^{m}+\mu_{-, i}^{m}\right) .
$$

We let $i_{o}$ be the first index for which $Q_{i_{o}}$ is in the nondegenerate regime, i.e. we choose $i_{o} \in \mathbb{N}_{0} \cup\{\infty\}$ in such a way that $\mu_{-, i_{o}}>\frac{1}{2} \mu_{+, i_{o}}$ and $\mu_{-, i} \leq \frac{1}{2} \mu_{+, i}$ for any $i<i_{o}$. If $\mu_{-, i} \leq \frac{1}{2} \mu_{+, i}$ for any $i \in \mathbb{N}_{0}$, we set $i_{o}=\infty$. We will apply Proposition 6.5 to prove by induction that

$$
Q_{i_{o}} \subset Q_{i_{o}-1} \subset \ldots \subset Q_{o}, \quad \text { and } \underset{Q_{i}}{\operatorname{Osc}} u^{m} \leq \omega_{i}^{m},
$$

for any $i \in\left\{0, \ldots, i_{o}\right\}$, (resp. $i \in \mathbb{N}_{0}$ if $i_{o}=\infty$ ). For $i=0$ the assertion (6.27) is a direct consequence of the definition of $\omega_{o}$. If $i_{o}=0$, we are finished and therefore it remains to consider the case where $i_{o}>0$. We now assume that (6.27) is satisfied for some $i \in\left\{0, \ldots, i_{o}-1\right\}$. Then, we have $\mu_{+, i}^{m}=\mu_{-, i}^{m}+\omega_{i}^{m} \geq \sup _{Q_{i}} u^{m}$ and hence $\mu_{+, i} \geq$ $\sup _{Q_{i}} u$. Moreover, we have $\sup _{Q_{i}} \psi^{m} \leq \frac{1}{2}\left(\mu_{+, i}^{m}+\mu_{-, i}^{m}\right)$ by $(6.26)$, respectively by (6.25). Therefore, we can apply Proposition 6.5 on the cylinders $Q_{i}$ to infer that $Q_{i+1} \subset Q_{i}$ and

$$
\underset{Q_{i+1}}{\operatorname{Osc}} u^{m} \leq \omega_{i+1}^{m}
$$

This proves the claim (6.27).

If $i_{o}<\infty$, we redefine the remaining cylinders so that Proposition 6.6 can be applied. Let $\theta_{*}:=\mu_{+, i_{o}}^{1-m} \leq \theta_{i_{o}}$ and redefine the cylinders for $i \in\left\{i_{o}+1, \ldots\right\}$ by

$$
Q_{i}:=Q_{\varrho_{i}, \theta_{*} \varrho_{i}^{2}}\left(z_{o}\right), \quad \text { with } \varrho_{i}:=\hat{\eta}^{i-i_{o}} \varrho_{i_{o}}, \hat{\eta}:=\sqrt{\frac{1}{32} \nu_{o}} .
$$

Note that $Q_{i_{o}} \supset Q_{i_{o}+1} \supset \ldots$ and that the redefinition of $Q_{i}$ also leads to a redefinition of $\omega_{i}, \mu_{-, i}$ and $\mu_{+, i}$. 
Our aim is to apply Proposition 6.6 on the cylinder $Q_{i_{o}}^{*}:=Q_{\varrho_{i_{o}}, \theta_{*} \varrho_{i_{o}}^{2}}\left(z_{o}\right) \subset Q_{i_{o}}$, with the parameters $\mu_{+, i_{o}}$ and $\mu_{-, i_{o}}$. To this end, we check that

$$
\inf _{Q_{i_{o}}^{*}} u \geq \inf _{Q_{i_{o}}} u=\mu_{-, i_{o}} \quad \text { and } \quad \sup _{Q_{i_{o}}^{*}} u \leq \sup _{Q_{i_{o}}} u \leq \mu_{+, i_{o}} .
$$

Moreover, from (6.26), respectively from (6.25), we know

$$
\sup _{Q_{i_{o}}^{*}} \psi^{m} \leq \sup _{Q_{i_{o}}} \psi^{m} \leq \frac{1}{2}\left(\mu_{+, i_{o}}+\mu_{-, i_{o}}\right),
$$

and the definition of $\omega_{i_{o}}$, respectively the choice of $\varrho_{o}$, implies

$$
\mu_{+, i_{o}}^{m}-\mu_{-, i_{o}}^{m}=\omega_{i_{o}}^{m} \geq 2 \underset{Q_{i_{o}}}{\operatorname{osc}} \psi^{m} \geq \underset{Q_{i_{o}}^{*}}{\operatorname{osc}} \psi^{m} .
$$

Hence, Proposition 6.6 is applicable on $Q_{i_{o}}^{*}$ and provides us with the estimate

$$
\underset{Q_{i}}{\operatorname{OSc}} u^{m} \leq \omega_{i}^{m}, \quad \text { for any } i \in\left\{i_{o}+1, \ldots\right\} .
$$

For $i \in \mathbb{N}_{0}$ we now define

$$
r_{i}:=\min \left\{1, \theta_{o}^{1 / 2}, \theta_{*}^{1 / 2}\right\} \varrho_{i}
$$

so that

$$
Q_{r_{i}}\left(z_{o}\right):=Q_{r_{i}, r_{i}^{2}}\left(z_{o}\right) \subset Q_{i}, \quad \text { for any } i \in \mathbb{N}_{0} .
$$

Therefore, for any $i \in \mathbb{N}_{0}$ we can use either (6.27), or (6.28) to conclude that

$$
\underset{Q_{r_{i}}}{\operatorname{OSc}} u^{m} \leq \underset{Q_{i}}{\operatorname{osc}} u^{m} \leq \omega_{i}^{m} \leq \delta \omega_{i-1}^{m}+2 \underset{Q_{i-1}}{\operatorname{osc}} \psi^{m} \leq \delta^{i} \omega_{o}^{m}+2 \sum_{j=0}^{i-1} \delta^{j} \underset{Q_{i-1-j}}{\operatorname{osc}} \psi^{m} .
$$

In the case $i-1-j \leq i_{o}$, we infer from assumption (6.1) that

$$
\begin{aligned}
\underset{Q_{i-1-j}}{\operatorname{Osc}} \psi^{m} & \leq c\left(\varrho_{i-1-j}^{\beta}+\left(\theta_{i-1-j} \varrho_{i-1-j}^{2}\right)^{\frac{\beta}{2}}\right)=c\left(1+\omega_{i-1-j}^{\frac{\beta(1-m)}{2}}\right) \varrho_{i-1-j}^{\beta} \\
& \leq c\left(1+\left(\delta^{\frac{i-1-j}{m}} \omega_{o}\right)^{\frac{\beta(1-m)}{2}}\right) \varrho_{i-1-j}^{\beta} \leq c\left(1+\omega_{o}^{\frac{\beta(1-m)}{2}}\right) \delta^{\frac{(i-1-j) \beta(1-m)}{2 m}} \varrho_{i-1-j}^{\beta} \\
& =c\left(1+\omega_{o}^{\frac{\beta(1-m)}{2}}\right)\left(\frac{\nu_{o}}{32}\right)^{\frac{\beta(i-1-j)}{2}} \varrho_{o}^{\beta} .
\end{aligned}
$$

For $i-1-j>i_{o}$ we get the same bound, since

$$
\begin{aligned}
\underset{Q_{i-1-j}}{\mathrm{OSc}} \psi^{m} & \leq c\left(\varrho_{i-1-j}^{\beta}+\left(\theta_{*} \varrho_{i-1-j}^{2}\right)^{\frac{\beta}{2}}\right) \leq c\left(1+\omega_{i_{o}}^{\frac{\beta(1-m)}{2}}\right) \varrho_{i-1-j}^{\beta} \\
& \leq c\left(1+\left(\delta^{\frac{i_{o}}{m}} \omega_{o}\right)^{\frac{\beta(1-m)}{2}}\right) \varrho_{i-1-j}^{\beta} \leq c\left(1+\omega_{o}^{\frac{\beta(1-m)}{2}}\right) \delta^{\frac{i_{o} \beta(1-m)}{2 m}} \varrho_{i-1-j}^{\beta} \\
& =c\left(1+\omega_{o}^{\frac{\beta(1-m)}{2}}\right)\left(\frac{\nu_{o}}{32}\right)^{\frac{\beta(i-1-j)}{2}} \varrho_{o}^{\beta} .
\end{aligned}
$$


Inserting this above, we conclude for any $i \in \mathbb{N}_{0}$ that

$$
\underset{Q_{r_{i}}}{\operatorname{OSc}} u^{m} \leq \delta^{i} \omega_{o}^{m}+c\left(1+\omega_{o}^{\frac{\beta(1-m)}{2}}\right) \varrho_{o}^{\beta} \sum_{j=0}^{i-1} \delta^{j}\left(\frac{\nu_{o}}{32}\right)^{\frac{\beta(i-1-j)}{2}} .
$$

With the abbreviation

$$
\varkappa:=\max \left\{\delta,\left(\frac{\nu_{o}}{32}\right)^{\frac{\beta}{2}}\right\}
$$

this shows that

$$
\underset{Q_{r_{i}}}{\operatorname{OSc}} u^{m} \leq \delta^{i} \omega_{o}^{m}+c i \varkappa^{i-1}\left(1+\omega_{o}^{\frac{\beta(1-m)}{2}}\right) \varrho_{o}^{\beta} .
$$

Since $i \sqrt{\varkappa}^{i} \leq-2 /(e \log \varkappa)$, this leads us to

$$
\underset{Q_{r_{i}}}{\operatorname{OSc}} u^{m} \leq \delta^{i} \omega_{o}^{m}+c \sqrt{\varkappa}^{i}\left(1+\omega_{o}^{\frac{\beta(1-m)}{2}}\right) \varrho_{o}^{\beta},
$$

for a constant $c$ depending only on $n, m, \beta$, and $[\psi]_{0 ; \beta, \beta / 2}$. Now, we define

$$
\gamma_{1}:=\min \left\{\frac{\log \varkappa}{2 \log \eta}, \gamma_{o}\right\}
$$

and note that $\gamma_{1} \leq \frac{\log \delta}{\log \eta}$. Therefore, from the last inequality and the fact $\eta^{i} \leq \frac{\varrho_{i}}{\varrho_{o}}$ we conclude that

$$
\begin{aligned}
\underset{Q_{r_{i}}}{\operatorname{OSc}} u^{m} & \leq \eta^{\gamma_{1} i} \omega_{o}^{m}+c \eta^{\gamma_{1} i}\left(1+\omega_{o}^{\frac{\beta(1-m)}{2}}\right) \varrho_{o}^{\beta} \\
& \leq c\left(n, m, \beta,[\psi]_{0 ; \beta, \beta / 2},\|u\|_{L^{\infty}}, R\right) r_{i}^{\gamma_{1}} .
\end{aligned}
$$

Now, we consider $r \in(0, R]$. If $r \in\left(0, r_{o}\right)$ we choose $i \in \mathbb{N}_{0}$ such that $r_{i+1} \leq r<r_{i}$. Then, from (6.29) we have

$$
\underset{Q_{r}}{\operatorname{OSc}} u^{m} \leq \underset{Q_{r_{i}}}{\operatorname{Oosc}} u^{m} \leq c r_{i}^{\gamma_{1}} \leq c \eta^{-\gamma_{1}} r^{\gamma_{1}} .
$$

Otherwise, if $r \in\left[r_{o}, \varrho_{o}\right)$, we estimate

$$
\underset{Q_{r}}{\operatorname{Osc}} u^{m} \leq \underset{Q_{\varrho_{o}, \varrho_{o}^{2-\varepsilon}}}{\operatorname{Osc}} u^{m} \leq 2 \Psi\left(\varrho_{o}\right) \leq c\left(\frac{r}{\varrho_{o}}\right)^{\gamma_{1}} \Psi\left(\varrho_{o}\right) \leq c r^{\gamma_{1}} .
$$

Finally, by the choice of $\varrho_{o}$ we have for $r \in\left[\varrho_{o}, R\right]$ that

$$
\underset{Q_{r}}{\operatorname{OSc}} u^{m} \leq \underset{Q_{r, r^{2-\varepsilon}}^{\mathrm{OSC}}}{\operatorname{or}} u^{m} \leq 2 \Psi(r) \leq c r^{\gamma_{o}} \leq c r^{\gamma_{1}} .
$$

Therefore, we conclude that $u^{m}$ is Hölder continuous at $z_{o}$. Since $z_{o} \in \Omega_{T}$ was arbitrary, this proves that $u^{m}$, and hence also $u$, is locally Hölder continuous in $\Omega_{T}$. This finishes the proof of Theorem 1.1. 


\section{References}

1. Alt, H. and Luckhaus, S., Quasilinear elliptic-parabolic differential equations, Math. Z. 183 (1983), 311-341.

2. Avelin, B. and LukKARI, T., A comparison principle for the porous medium equation and its consequences, submitted, available at arXiv: 1505.07579.

3. Bögelein, V., DuzaAr, F. and Gianazza, U., Continuity estimates for porous medium type equations with measure data, J. Funct. Anal. 267 (2014), 33513396.

4. Bögelein, V., Duzaar, F. and Mingione, G., Degenerate problems with irregular obstacles, J. Reine Angew. Math. 650 (2011), 107-160.

5. Bögelein, V., Lukkari, T. and Scheven, C., The obstacle problem for the porous medium equation, Math. Ann. 363 (2015), 455-499.

6. Bögelein, V. and Scheven, C., Higher integrability in parabolic obstacle problems, Forum Math. 24 (2012), 931-972.

7. Daskalopoulos, P. and Kenig, C. E., Degenerate Diffusions - Initial Value Problems and Local Regularity Theory, European Mathematical Society (EMS), Zürich, 2007.

8. DiBenedetto, E., Degenerate Parabolic Equations, Springer Universitext, Springer, New York, 1993.

9. DiBenedetto, E., Gianazza, U. and Vespri, V., Harnack's Inequality for Degenerate and Singular Parabolic Equations, Springer Monographs in Mathematics, Springer, New York, 2012.

10. DiBenedetto, E. and Friedman, A., Hölder estimates for nonlinear degenerate parabolic systems, J. Reine Angew. Math. 357 (1985), 1-22.

11. Giusti, E., Direct Methods in the Calculus of Variations, World Scientific, Singapore, 2003.

12. Korte, R., Kuusi, T. and Siljander, J., Obstacle problem for nonlinear parabolic equations, J. Differential Equations 246 (2009), 3668-3680.

13. Kuusi, T., Mingione, G. and Nyström, K., Sharp regularity for evolutionary obstacle problems, interpolative geometries and removable sets, J. Math. Pures Appl. (9) 101 (2014), 119-151.

14. Lindqvist, P. and Parviainen, M., Irregular time dependent obstacles, J. Funct. Anal. 263 (2012), 2458-2482.

15. Lions, J.-L., Quelques méthodes de résolution des problèmes aux limites non linéaires, Dunod, Paris, 1969.

16. Naumann, J., Einführung in die Theorie parabolischer Variationsungleichungen, Teubner-Texte zur Mathematik 64, BSB B. G. Teubner Verlagsgesellschaft, Leipzig, 1984.

17. VÁzquez, J. L., The Porous Medium Equation - Mathematical Theory, Oxford University Press, Oxford, 2007.

18. Wu, Z., ZhaO, J., Yin, J. and Li, H., Nonlinear Diffusion Equations, World Scientific Publishing Co., Inc., River Edge, NJ, 2001. 
Verena Bögelein

Fachbereich Mathematik

Universität Salzburg

Hellbrunner Str. 34

AT-5020, Salzburg

Austria

verena.boegelein@sbg.ac.at

Teemu Lukkari

Department of Mathematics and System

Analysis

P.O. Box 11100

FI-00076 Aalto University

Espoo

Finland

teemu.lukkari@aalto.fi

Received September 1, 2015
Christoph Scheven

Fakultät für Mathematik

Universität Duisburg-Essen

Thea-Leymann-Str. 9

DE-45127 Essen

Germany

christoph.scheven@uni-due.de 